Georgian Mathematical Journal

Volume 8 (2001), Number 4, 823-844

\title{
ON HOMOMORPHISMS, OPEN OPERATORS AND THEIR ADJOINTS
}

\author{
D. ZARNADZE
}

\begin{abstract}
The well-known A. Grothendieck's theorem on a homomorphism between locally convex spaces is generalized to the case of topologies which are incompatible with dualities. On the basis of this theorem, necessary and sufficient conditions are obtained for a weak homomorphism (resp. its adjoint operator, resp. its double adjoint operator) to be again a homomorphism in various topologies of the initial (resp. dual, resp. bidual) spaces. Some new classes of pairs of locally convex spaces satisfying these conditions are established. The results obtained have enabled us to reveal new properties of frequently encountered homomorphisms and weakly open operators, as well as to strengthen and generalize some well-known results.
\end{abstract}

2000 Mathematics Subject Classification: 46A03, 47A05.

Key words and phrases: Homomorphism, locally convex space, dual space, bidual space, adjoint operator, double adjoint operator, open operator.

\section{INTRODUCTION}

In this paper we investigate homomorphisms, i.e., linear, continuous and open operators $A: E \rightarrow F$ which map arbitrary Hausdorff, locally convex spaces (briefly, LCS) $E$ into the same spaces $F$ and their adjoint mappings. We study the question whether a change of the topologies of $E$ and $F$ affects the stability of homomorphisms. Such problems frequently arise in applications and have been intensively studied since the days of S. Banach for various spaces and topologies. The most important results were obtained in J. Dieudonné [1], J. Dieudonné and L. Schwartz [2], A. Grothendieck [3], G. Köthe [4], [5], F. Browder [6], V. S. Retakh [7], V. P. Palamodov [8], K. Floret and V. B. Moscatelli [9]. The most complete account of the results known so far in this area is given in the monographs [10]-[12] though, it seems to us, in a somewhat incoherent manner.

We attach special importance to studying strong homomorphisms and their strong adjoints, i.e., cases in which the initial and dual spaces are equipped with strong topologies. The need to investigate them was raised in [2], §12. In this connection, two examples of homomorphisms of Fréchet spaces whose adjoints are not strong homomorphisms were constructed in [3], where the dual spaces are equipped with strong topologies. In particular, these examples are

ISSN 1072-947X / \$8.00 / (c) Heldermann Verlag www.heldermann.de 
meticulously studied in $\S 4$ (see Examples 1 and 2), where the initial and dual spaces are equipped with various topologies. We are also well familiar with the following A. Grothendieck's theorem on a homomorphism [10, p. 8], which gives a necessary and sufficient condition for a weak homomorphism to be also a homomorphism.

Let $\left(E, \mathfrak{T}_{1}\right)$ and $\left(F, \mathfrak{T}_{2}\right)$ be LCS, $\mathfrak{M}_{1}$ and $\mathfrak{M}_{2}$ the classes of equicontinuous subsets in $E^{\prime}$ and $F^{\prime}$, respectively. A weak homomorphism $A$ of the space $\left(E, \sigma\left(E, E^{\prime}\right)\right)$ in the space $\left(F, \sigma\left(F, F^{\prime}\right)\right)$ is a homomorphism of the space $\left(E, \mathfrak{T}_{1}\right)$ in the space $\left(F, \mathfrak{T}_{2}\right)$ if and only if $A^{\prime}\left(\mathfrak{M}_{2}\right)=\mathfrak{M}_{1} \cap A^{\prime}\left(F^{\prime}\right)$, where $A^{\prime}$ is the adjoint operator and $\mathfrak{M}_{1} \cap A^{\prime}\left(F^{\prime}\right)=\left\{M \in \mathfrak{M}_{1} ; M \subset A^{\prime}\left(F^{\prime}\right)\right\}$.

This theorem however does not hold for topologies which are incompatible with the dualities $\left\langle E, E^{\prime}\right\rangle$ and $\left\langle F, F^{\prime}\right\rangle$. In particular, the theorem fails to be valid for the uniform convergence topologies $\mathfrak{T}_{1}=\mathfrak{T}_{\mathfrak{M}_{1}}$ and $\mathfrak{T}_{2}=\mathfrak{T}_{\mathfrak{M}_{2}}$, where $\mathfrak{M}_{1}$ and $\mathfrak{M}_{2}$ are the classes of weakly bounded subsets $E^{\prime}$ and $F^{\prime}$, respectively. Namely, it does not hold for strong topologies when $\mathfrak{M}_{1}$ are $\mathfrak{M}_{2}$ are the families of all weakly bounded sets in $E^{\prime}$ and $F^{\prime}$, respectively. The counterexample given in [10, p. 10], for the latter case shows that there exists a weak homomorphism $K$ of the LCS $E$ on the same space $F$, for which $K^{\prime}\left(\mathfrak{M}_{2}\right)=K^{\prime}\left(F^{\prime}\right) \cap \mathfrak{M}_{1}$, but $K$ is not a strong homomorphism. Difficulties which one encounters in the investigation of strong homomorphisms are also mentioned in [10, p. 10]. This explains why no necessary and sufficient condition has actually been so far found for a weak homomorphism to be also a strong homomorphism and, generally, a homomorphism in topologies which are incompatible with the dualities $\left\langle E, E^{\prime}\right\rangle$ and $\left\langle F, F^{\prime}\right\rangle$.

In [13-14], while investigating the dual characterization of quojections (strictly regular Fréchet spaces), it was proved that the adjoint operator to the homomorphism of a quojection on the Banach space is a strong homomorphism (contrary to Example 1 in $\S 4$ ). Analysis of these results and the above-mentioned examples has enabled us to prove a generalization of A. Grothendieck's theorem. Our approach is the unified one and based on the statement that in different situations the homomorphy of $A$ (resp. $A^{\prime}$, resp. $A^{\prime \prime}$ ) is characterized by the coincidence of two naturally generated topologies on the quotient space $E / \operatorname{Ker} A$ (resp. $F^{\prime} / \operatorname{Ker} A^{\prime}$, resp. $\left.E^{\prime \prime} / \operatorname{Ker} A^{\prime \prime}\right)$ and on the range $A(E)$ (resp. $A^{\prime}\left(F^{\prime}\right)$, resp. $\left.A^{\prime \prime}\left(F^{\prime \prime}\right)\right)$.

In $\S 1$ of this paper, a generalization of A. Grothendieck's theorem is proved for topologies which in particular are incompatible with dualities. Hence we obtain necessary and sufficient conditions for the weak homomorphism $A$ to be again a homomorphism when spaces are equipped with strong topologies, Mackey topologies and topologies of strong precompact convergence. Using a similar reasoning, a necessary and sufficient condition is obtained for a weak homomorphism to be also a homomorphism when spaces are equipped with associated bornological topologies. Classes of pairs of the LCS $E$ and $F$ are found, for which an arbitrary weak homomorphism is again a homomorphism in the above-mentioned topologies of the initial spaces. In particular, strong 
homomorphisms are investigated with sufficient completeness, and some wellknown results are strengthened and generalized. Applying the results from [6], we also obtain conditions for weakly open operators with closed graphs to be open and strongly open.

In $\S 2$, a necessary and sufficient condition is proved for the adjoint operator to a weak homomorphism to be again a homomorphism when the dual spaces are equipped with uniform convergence topologies on the subsets of classes of bounded sets of the initial spaces. An analogous result is also proved when the dual spaces are equipped with the so-called inductive topologies. Such a topology in the dual space was introduced in [5] as the strongest locally convex topology which preserves the boundedness of equicontinuous sets. The adjoint operators to homomorphisms were considered in [7], [8] and [16] when the dual spaces are equipped with inductive topologies.

In $\S 3$, double adjoints to homomorphisms are studied when bidual spaces are equipped with weak, strong and natural topologies. Necessary and sufficient conditions are given for the double adjoint operator $A^{\prime \prime}$ to the weak homomorphism $A$ to be a homomorphism in the above-mentioned topologies depending on whether $A^{\prime}$ is a strong homomorphism.

In $\S 4$, applications of the results obtained in the preceding sections to the known homomorphisms are given. Also, their new properties are established, which show that our proven theorems can successfully be used to investigate homomorphisms and their adjoint mappings.

We should make some necessary clarification of the terms and notation which are borrowed mainly from [11], [17].

A linear operator $A: E \rightarrow F$, mapping the LCS $E$ into the LCS $F$ is called open if for any open set $U$ whose image $A(U)$ is an open subset in $A(E)$ (in the topology induced by $F$ ). A linear continuous and open operator $A$ is called a topological homomorphism (or simply a homomorphism when this does not cause confusion). If a homomorphism is one-to-one (injective), then it is called a monomorphism.

Let $E$ be a LCS and $\mathfrak{M}$ be some class of weakly bounded subsets of the dual space containing the class of all finite sets $\mathfrak{F}$. By $\mathfrak{T}_{\mathfrak{M}}\left(E^{\prime}\right)$ we denote the topology on $E$ which is of uniform convergence on the sets from $\mathfrak{M}$. In particular, by $\beta\left(E, E^{\prime}\right)$ (resp. $\sigma\left(E, E^{\prime}\right)$, resp. $\tau\left(E, E^{\prime}\right)$, resp. $\left.\mathfrak{T}_{c}\left(E^{\prime}\right)\right)$ we denote the topology on $E$ which is of uniform convergence on all weakly bounded (resp. finite, resp. absolutely convex and $\sigma\left(E^{\prime}, E\right)$-compact, resp. strongly precompact) sets from $E^{\prime}$. The topology $\tau\left(E, E^{\prime}\right)$ is called the Mackey topology of the dual pair $\left\langle E, E^{\prime}\right\rangle$. The space $E$ equipped with the Mackey topology is called the Mackey space. The topologies of uniform convergence $\beta\left(E^{\prime}, E\right), \sigma\left(E^{\prime}, E\right), \tau\left(E^{\prime}, E\right)$ and $\mathfrak{T}_{c}(E)$ on $E^{\prime}$ are defined symmetrically. The strong bidual space, i.e., the strong dual space to the strong dual space $\left(E^{\prime}, \beta\left(E^{\prime}, E\right)\right)$ is denoted by $\left(E^{\prime \prime}, \beta\left(E^{\prime \prime}, E^{\prime}\right)\right)$. $\operatorname{A~LCS~}(E, \mathfrak{T})$ is called reflexive if $E=E^{\prime \prime}$ and $\mathfrak{T}=\beta\left(E^{\prime \prime}, E^{\prime}\right)$. For the subspace $G$ of the LCS $\left(E, \mathfrak{T}_{\mathfrak{M}}\left(E^{\prime}\right)\right)$ we denote by $\mathfrak{T}_{\mathfrak{M}}\left(E^{\prime}\right) \cap G$ the topology induced on $G$, while by $\mathfrak{T}_{\widehat{M}}\left(E^{\prime} / G^{\perp}\right)$ the uniform convergence topology on the class 
$\widehat{\mathfrak{M}}=K(\mathfrak{M})=\{K(M) ; M \in \mathfrak{M}\}$, where $K$ is the canonical mapping of $E^{\prime}$ on $E^{\prime} / G^{\perp}$. Further, for the closed subspace $G$ of the space $\left(E, \mathfrak{T}_{\mathfrak{M}}\left(E^{\prime}\right)\right)$ we denote by $\mathfrak{T}_{\mathfrak{M}}\left(E^{\prime}\right) / G$ the quotient topology on $E / G$, while by $\mathfrak{T}_{\overline{\mathfrak{M}}}\left(G^{\perp}\right)$ the topology on $E / G$ of uniform convergence on the class $\overline{\mathfrak{M}}=\left\{M \in \mathfrak{M} ; M \subset G^{\perp}\right\} \subset \mathfrak{M}$.

It is well-known that the Fréchet space with a generating increasing sequence of semi-norms $\left\{p_{n}\right\}$ is isomorphic to the projective limit of a sequence of Banach spaces $E_{n}=\left(E / \widehat{\operatorname{Ker} p_{n}}, \widehat{p_{n}}\right)$ with respect to the mappings $\bar{\pi}_{n m}: E_{m} \rightarrow E_{n}(n \leq$ $m)$, where $E_{n}$ is a completion of the normed space $\left(E / \operatorname{Ker} p_{n}, \widehat{p_{n}}\right)$ and $\bar{\pi}_{n m}$ is the continuation, on $E_{m}$, of the canonical mapping $\pi_{n m}: E / \operatorname{Ker} p_{m} \rightarrow E / \operatorname{Ker} p_{n}$. The Fréchet space is called the quojection if it is isomorphic to the projective limit of a sequence of Banach spaces with respect to surjective mappings. This means that the mappings $\pi_{n m}(n \leq m)$ are homomorphisms.

The strong adjoint of the quojection $E$ is a strong $(L B)$-space which is represented as $\left(E^{\prime}, \beta\left(E^{\prime}, E\right)\right)=s \cdot \lim E_{n}^{\prime}$, i.e., the class of quojections coincides with the class of strictly regular Fréchet spaces [18]. A Fréchet space is called a prequojection [19] if its strongly bidual space is a quojection. As is known [20], the class of prequojections coincides with the class of Fréchet spaces whose strong dual space is any strict $(L B)$-space.

\section{On Homomorphisms Between Locally Convext Spaces}

In this section, conditions are given for a homomorphism between the LCS $E$ and $F$ to be again a homomorphism in various known topologies of the spaces $E$ and $F$. Let $A=J \breve{A} K$ be the natural decomposition of the weak homomorphism $A$, where $K$ is a canonical homomorphism of spaces $\left(E, \sigma\left(E, E^{\prime}\right)\right)$ on $\left(E / \operatorname{Ker} A, \sigma\left(E, E^{\prime}\right) / \operatorname{Ker} A\right), \check{A}$ is the weak isomorphism $\left(E / \operatorname{Ker} A, \sigma\left(E, E^{\prime}\right) /\right.$ $\operatorname{Ker} A)$ on $\left(A(E), \sigma\left(F, F^{\prime}\right) \cap A(E)\right)$ and $J$ is the monomorphism $\left(A(E), \sigma\left(F, F^{\prime}\right) \cap\right.$ $A(E))$ in $\left(F, \sigma\left(F, F^{\prime}\right)\right)$. By virtue of the known properties of a weak topology $\left(\left[21\right.\right.$, p. 276]) we have that the equalities $\sigma\left(E, E^{\prime}\right) / \operatorname{Ker} A=\sigma(E / \operatorname{Ker} A$, $\operatorname{Ker} A^{\perp}$ ) on $E / \operatorname{Ker} A$ and $\sigma\left(F, F^{\prime}\right) \cap A(E)=\sigma\left(A(E), F^{\prime} / A(E)^{\perp}\right)$, are valid on $A(E)$ because $(E / \operatorname{Ker} A)^{\prime}=\operatorname{Ker} A^{\perp}$ and $A(E)^{\prime}=F^{\prime} / A(E)^{\perp}$. Therefore the spaces $\left(E / \operatorname{Ker} A, \sigma\left(E / \operatorname{Ker} A, \operatorname{Ker} A^{\perp}\right)\right)$ and $\left.A(E), \sigma\left(A(E), F^{\prime} / A(E)^{\perp}\right)\right)$ are isomorphic. Hence it follows that the spaces $\left((E / \operatorname{Ker} A)^{\prime}, \sigma\left((E / \operatorname{Ker} A)^{\prime}, E /\right.\right.$ $\operatorname{Ker} A)$ ) and $\left(A(E)^{\prime}, \sigma\left(A(E)^{\prime}, A(E)\right)\right)$ too are weakly isomorphic and this isomorphism is realized by the mapping $\check{A}^{\prime}$ adjoint to the mapping $\check{A}$. Denote by $\widehat{\mathfrak{M}_{2}}$ the class $K_{1}\left(\mathfrak{M}_{2}\right)=\left\{K_{1}(M) ; M \in \mathfrak{M}_{2}\right\}$, where $K_{1}: F^{\prime} \rightarrow F^{\prime} / A(E)^{\perp}$ is a canonical mapping. Let $\overline{\mathfrak{M}_{2}}$ denote the family $\check{A}^{\prime}\left(\widehat{\mathfrak{M}}_{2}\right)$. Since $\check{A}^{\prime}$ is the weak

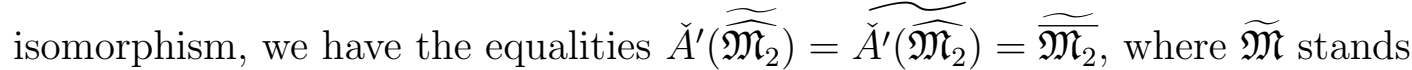
for the saturated cover of the class $\mathfrak{M}$.

Theorem 1. Let $E$ and $F$ be the locally convex spaces with the saturated classes of weakly bounded subsets $\mathfrak{M}_{1}$ and $\mathfrak{M}_{2}$ of the dual spaces $E^{\prime}$ and $F^{\prime}$, respectively. The weak homomorphism $A$ of the space $E$ in the space $F$ is the homomorphism of the space $\left(E, \mathfrak{T}_{\mathfrak{M}_{1}}\left(E^{\prime}\right)\right)$ in the space $\left(F, \mathfrak{T}_{\mathfrak{M}_{2}}\left(F^{\prime}\right)\right)$ if and only if the following conditions are satisfied: 
a) $\overline{\mathfrak{M}_{1}}=\widetilde{\overline{\mathfrak{M}_{2}}}$ and $A^{\prime}\left(\mathfrak{M}_{2}\right) \subset \mathfrak{M}_{1}$, where $\overline{\mathfrak{M}_{1}}=\left\{M \in \mathfrak{M}_{1} ; M \subset \operatorname{Ker} A^{\perp}\right\}$;

b) $\mathfrak{T}_{\mathfrak{M}_{1}}\left(E^{\prime}\right) / \operatorname{Ker} A=\mathfrak{T}_{\overline{\mathfrak{M}}_{1}}\left(\operatorname{Ker} A^{\perp}\right)$ on $E / \operatorname{Ker} A$, where $\mathfrak{T}_{\overline{\mathfrak{M}}_{1}}\left(\operatorname{Ker} A^{\perp}\right)$ is a topology of uniform convergence on the class $\overline{\mathfrak{M}}_{1}$;

c) $\mathfrak{T}_{\mathfrak{M}_{2}}\left(F^{\prime}\right) \cap A(E)=\mathfrak{T}_{\widehat{\mathfrak{M}}_{2}}\left(F^{\prime} / A(E)^{\perp}\right)$ on $A(E)$, where $\mathfrak{T}_{\widehat{\mathfrak{M}}_{2}}\left(F^{\prime} / A(E)^{\perp}\right)$ is the uniform convergence topology on $\widehat{\mathfrak{M}}_{2}$.

Proof. Sufficiency. The condition a) implies that the spaces (E/Ker $A$, $\left.\mathfrak{T}_{\overline{\mathfrak{M}}_{1}}\left(\operatorname{Ker} A^{\perp}\right)\right)$ and $\left(A(E), \mathfrak{T}_{\widehat{\mathfrak{M}}_{2}}\left(F^{\prime} / A(E)^{\perp}\right)\right)$ are isomorphic, where $\mathfrak{T}_{\overline{\mathfrak{M}}_{1}}\left(\operatorname{Ker} A^{\perp}\right)$ is the topology, on $E / \operatorname{Ker} A$, of uniform convergence on $\overline{\mathfrak{M}}_{1}$, while $\mathfrak{T}_{\widehat{\mathfrak{M}}_{2}}\left(F^{\prime} /\right.$ $\left.A(E)^{\perp}\right)$ is the topology, on $A(E)$, of uniform convergence on the sets $K_{1}\left(\mathfrak{M}_{2}\right) \subset$ $F^{\prime} / A(E)^{\perp}$. By virtue of Theorem 1 ([10, p. 3]) the conditions $A^{\prime}\left(\mathfrak{M}_{2}\right) \subset \mathfrak{M}_{1}$ imply the continuity of the operator $A$ from the space $\left(E, \mathfrak{T}_{\mathfrak{M}_{1}}\left(E^{\prime}\right)\right)$ into $\left(F, \mathfrak{T}_{\mathfrak{M}_{2}}\left(F^{\prime}\right)\right)$. Furthermore, the conditions b) and c) imply that $\check{A}$ is also the isomorphism of the spaces $\left(E / \operatorname{Ker} A, \mathfrak{T}_{\mathfrak{M}_{1}}\left(E^{\prime}\right) / \operatorname{Ker} A\right)$ and $\left(A(E), \mathfrak{T}_{\mathfrak{M}_{2}}\left(F^{\prime}\right) \cap\right.$ $A(E))$, i.e., $A$ is the homomorphism of the space $\left(E, \mathfrak{T}_{\mathfrak{M}_{1}}\left(E^{\prime}\right)\right)$ in $\left(F, \mathfrak{T}_{\mathfrak{M}_{2}}\left(F^{\prime}\right)\right)$.

Necessity. Let the weak homomorphism $A$ of the space $E$ in $F$ be also the homomorphism of the space $\left(E, \mathfrak{T}_{\mathfrak{M}_{1}}\left(E^{\prime}\right)\right)$ in $\left(F, \mathfrak{T}_{\mathfrak{M}_{2}}\left(F^{\prime}\right)\right)$. As said above, $\check{A}$ is the weak isomorphism of the space $\left(E / \operatorname{Ker} A, \sigma\left(E / \operatorname{Ker} A, \operatorname{Ker} A^{\perp}\right)\right)$ onto $\left(A(E), \quad \sigma\left(A(E), \quad F^{\prime} / A(E)^{\perp}\right)\right)$. In these spaces the topologies $\mathfrak{T}_{\mathfrak{M}_{1}}\left(E^{\prime}\right) / \operatorname{Ker} A$ and $\mathfrak{T}_{\mathfrak{M}_{2}}\left(F^{\prime}\right) \cap A(E)$ also coincide since $A$ is the homomorphism of the space $\left(E, \mathfrak{T}_{\mathfrak{M}_{1}}\left(E^{\prime}\right)\right)$ in $\left(F, \mathfrak{T}_{\mathfrak{M}_{2}}\left(F^{\prime}\right)\right)$. Hence, again by virtue of Theorem 1 from [10, p. 3], we obtain $A^{\prime}\left(\mathfrak{M}_{2}\right) \subset \mathfrak{M}_{1}$. This is equivalent to that the mapping $\check{A}$ is continuous since it is the mapping of the space $\left(E / \operatorname{Ker} A, \mathfrak{T}_{\overline{\mathfrak{M}}_{1}}\left(\operatorname{Ker} A^{\perp}\right)\right.$ ) onto $\left(A(E), \mathfrak{T}_{\widehat{\mathfrak{M}_{2}}}\left(F^{\prime} / A(E)^{\perp}\right)\right)$. Indeed, $A^{\prime}=K^{\prime} \check{A}^{\prime} J^{\prime}$, where $K^{\prime}, \check{A}^{\prime}$ and $J^{\prime}$ are the adjoints to the mappings $K, \check{A}$ and $J$, respectively. In that case we have $K^{\prime} \check{A}^{\prime} J^{\prime}\left(\mathfrak{M}_{2}\right) \subset \mathfrak{M}_{1}$, but, as is known, $J^{\prime}\left(\mathfrak{M}_{2}\right)=K_{1}\left(\mathfrak{M}_{2}\right)=\widehat{\mathfrak{M}_{2}}$ and therefore $K^{\prime} \check{A}^{\prime}\left(\widehat{\mathfrak{M}}_{2}\right) \subset \mathfrak{M}_{1}$, i.e., $\check{A}^{\prime}\left(\widehat{\mathfrak{M}}_{2}\right) \subset K^{\prime}(-1)\left(\mathfrak{M}_{1}\right)=\mathfrak{M}_{1} \cap$ Ker $A^{\perp}=\overline{\mathfrak{M}}_{1}$. Further, by virtue of Theorem 1 from $\left[21\right.$, p.276] we obtain the inequality $\mathfrak{T}_{\mathfrak{M}_{2}}\left(F^{\prime}\right) \cap A(E) \leq$ $\mathfrak{T}_{\overline{\mathfrak{M}}_{2}}\left(F^{\prime} / A(E)^{\perp}\right)$ on $A(E)$. Indeed, the equalities $K_{1}(M)^{0 A(E)}=J^{\prime}(M)^{0 A(E)}=$ $J^{-1}\left(M^{0 F}\right)=M^{0 F} \cap A(E)$ are valid for $M \in \mathfrak{M}_{2}$. The above inequality is true since in the saturated cover of $\widehat{\mathfrak{M}}_{2}$ there may occur sets not contained in the $K_{1}$-image of the sets from $\mathfrak{M}_{2}$. The inequality $\mathfrak{T}_{\overline{\mathfrak{M}}_{1}}\left(\operatorname{Ker} A^{\perp}\right) \leq \mathfrak{T}_{\mathfrak{M}_{1}}\left(E^{\prime}\right) / \operatorname{Ker} A$ can be proved in a similar manner using Theorem 3 from [21, p. 277]. On account of the preceding arguments the following diagram is valid:

$$
\begin{gathered}
\left(E / \operatorname{Ker} A, \mathfrak{T}_{\mathfrak{M}_{1}}\left(E^{\prime}\right) / \operatorname{Ker} A\right) \stackrel{\stackrel{\check{A}}{\rightleftarrows}}{\rightleftarrows}\left(A(E), \underset{\uparrow}{\uparrow} \mathfrak{T}_{\mathfrak{M}_{2}}\left(F^{\prime}\right) \cap A(E)\right) \\
\downarrow \\
\left(E / \operatorname{Ker} A, \mathfrak{T}_{\overline{\mathfrak{M}}_{1}}\left(\operatorname{Ker} A^{\perp}\right)\right) \stackrel{\check{A}}{\rightarrow}\left(A(E), \widetilde{T}_{\widehat{\mathfrak{M}}_{2}}\left(F^{\prime} / A(E)^{\perp}\right)\right.
\end{gathered}
$$

where the vertical arrows denote the continuous identical algebraic isomorphisms. Therefore the above two topologies on $E / \operatorname{Ker} A$ and $A(E)$ coincide. Furthermore, the topologies $\mathfrak{T}_{\overline{\mathfrak{M}}_{1}}\left(\operatorname{Ker} A^{\perp}\right)$ and $\mathfrak{T}_{\widehat{\mathfrak{M}}_{2}}\left(F^{\prime} / A(E)^{\perp}\right)$ on the weakly isomorphic spaces $E / \operatorname{Ker} A$ and $A(E)$ also coincide. By Theorem 4 ([21, p. 
$256])$, the saturated covers of the classes $\check{A}^{\prime}\left(\widehat{\mathfrak{M}}_{2}\right)$ and $\overline{\mathfrak{M}}_{1}$ also coincide, i.e., the condition a) too is fulfilled.

Note that if the classes $\mathfrak{M}_{1}$ and $\mathfrak{M}_{2}$ consist only of equicontinuous sets, then the class $\widehat{\mathfrak{M}}_{2}$ is also saturated and, the conditions b) and c) are always fulfilled by virtue of Theorems 1 and 2 ([21, pp. 275-276]). As the above-mentioned example from [21] shows, we do not have the same situation in the general case. Below we will give examples of the topologies for which the conditions b) and c) imply a).

Corollary. Let in the notation of Theorem $1 A$ be a weak monomorphism $E$ in F. A is a monomorphism of $\left(E, \mathfrak{T}_{\mathfrak{M}_{1}}\right)$ in $\left(F, \mathfrak{T}_{\mathfrak{M}_{2}}\right)$ if and only if $\overline{A^{\prime}\left(\mathfrak{M}_{2}\right)}=$ $\mathfrak{M}_{1}$, where $\widetilde{A^{\prime}\left(\mathfrak{M}_{2}\right)}$ consists of weak closures of sets of the form $A^{\prime}(M), M \in$ $\mathfrak{M}_{2}$.

This results gives a characterization of a monomorphism in the general case and differs from Theorem 4 in $[10$, p. 10] in that it requires that in the a priori conditions $A$ be a weak monomorphism.

Let us now give a concrete reformulation of Theorem 1 for the known topologies.

Theorem 2. Let $A$ be a weak homomorphism of the LCS E in the LCS F. A is a strong homomorphism, i.e., the homomorphism of the space $\left(E, \beta\left(E, E^{\prime}\right)\right)$ in the space $\left(F, \beta\left(F, F^{\prime}\right)\right)$ if and only if $\beta\left(E, E^{\prime}\right) / \operatorname{Ker} A=\beta\left(E / \operatorname{Ker} A, \operatorname{Ker} A^{\perp}\right)$ on $E / \operatorname{Ker} A$ and $\beta\left(F, F^{\prime}\right) \cap A(E)=\beta\left(A(E), F^{\prime} / A(E)^{\perp}\right)$ on $A(E)$, where $\beta\left(F, F^{\prime}\right) / \operatorname{Ker} A$ is the quotient topology of the strong topology $\beta\left(E, E^{\prime}\right), \beta\left(F, F^{\prime}\right) \cap$ $A(E)$ is the induced topology on $A(E)$, while $\beta\left(E / \operatorname{Ker} A, \operatorname{Ker} A^{\perp}\right)$ and $\beta((A(E)$, $\left.F^{\prime} / A(E)^{\perp}\right)$ - are the strong topologies of the dual pairs $\left\langle E / \operatorname{Ker} A, \operatorname{Ker} A^{\perp}\right\rangle$ and $\left\langle A(E), F^{\prime} / A(E)^{\perp}\right\rangle$, respectively.

One can prove this theorem by repeating the arguments used in proving Theorem 1, taking into account the fact that weakly continuous mappings are strongly continuous.

Note that in proving the necessity part of Theorem 2, the a priori conditions imposed on $A$ can be made weaker. Namely, for the mentioned topologies to coincide on $E / \operatorname{Ker} A$ and $A(E)$ it is sufficient to assume that $A$ is weakly continuous strong homomorphism. At that, one should not expect $A$ to be a weak homomorphism.

Corollary. If $A$ is a homomorphism of the barrelled $L C S\left(E, \mathfrak{T}_{1}\right)$ in the LCS $\left(F, \mathfrak{T}_{2}\right)$, then $A$ is a strong homomorphism.

Denote by $\mathfrak{T}_{b^{*}}\left(E^{\prime}\right)$ the topology, on $E$, of uniform convergence on strongly bounded sets from $E^{\prime}$, i.e., the uniform convergence topology on the bounded sets of the space $\left(E^{\prime}, \beta\left(E, E^{\prime}\right)\right)$.

Theorem 3. Let $A$ be a weak homomorphism of the LCS E in the LCS F. $A$ is a $\mathfrak{T}_{b^{*}}$-homomorphism, i.e., the homomorhism of the $\operatorname{LCS}\left(E, \mathfrak{T}_{b^{*}}\left(E^{\prime}\right)\right)$ in 
the $\operatorname{LCS}\left(F, \mathfrak{T}_{b^{*}}\left(F^{\prime}\right)\right)$ if and only if $\mathfrak{T}_{b^{*}}\left(E^{\prime}\right) / \operatorname{Ker} A=\mathfrak{T}_{b^{*}}\left(\operatorname{Ker} A^{\perp}\right)$ on $E / \operatorname{Ker} A$ and $\mathfrak{T}_{b^{*}}\left(F^{\prime}\right) \cap A(E)=\mathfrak{T}_{b^{*}}\left(F^{\prime} / A(E)^{\perp}\right)$ on $A(E)$.

Corollary. If $A$ is a homomorphism of the quasibarrelled LCS E in the LCS $F$, then $A$ is a $\mathfrak{T}_{b^{*}}$-homomorphism.

F. Browder studied in [6] open operators with a closed graph and called them again homomorhisms. He we will apply our results to obtain conditions for a weakly open operator to become strongly open, i.e., conditions under which the implication $e) \Rightarrow d$ ) in Theorem 2.1 from [6] holds true.

Theorem 4. Let $T: E \rightarrow F$ be a weakly open operator of the $L C S\left(E, \mathfrak{T}_{1}\right)$ in the LCS $\left(F, \mathfrak{T}_{2}\right)$ with a dense domain of definition $D(T)$ and closed kernel $\operatorname{Ker} T . T$ is strongly open if the following conditions are satisfied:

a) the topologies $\left(\beta\left(E, E^{\prime}\right) \cap D(T)\right) / \operatorname{Ker} T$ and $\beta\left(D(T) / \operatorname{Ker} T, \operatorname{Ker} T^{\perp}\right)$ coincide on the quotient space $D(T) / \operatorname{Ker} T$;

b) the topologies $\beta\left(F, F^{\prime}\right) \cap R(T)$ and $\beta\left(R(T), F^{\prime} / R(T)^{\perp}\right)$ coincide on the quotient space $R(T)$.

Proof. As is well known, $T$ is weakly open if and only if in its natural decomposition the linear bijection $\check{T}:\left(D(T) / \operatorname{Ker} T, \sigma\left(E, E^{\prime}\right) \cap D(T) / \operatorname{Ker} T\right) \rightarrow$ $\left(R(T), \sigma\left(F, F^{\prime}\right) \cap R(T)\right)$ is weakly open. The topologies $\sigma\left(F, F^{\prime}\right) \cap D(T) / \operatorname{Ker} T$ and $\sigma\left(D(T) / \operatorname{Ker} T, \operatorname{Ker} T^{\perp}\right)$ coincide by virtue of the properties of a weak topology on the quotient space $D(T) / \operatorname{Ker} T$ just like the topologies $\sigma\left(F, F^{\prime}\right) \cap R(T)$ and $\sigma\left(R(T), F^{\prime} / R(T)^{\perp}\right)$ coincide on $R(T)$. Therefore $\check{T}$ is weakly open as the mapping

$$
\check{T}:\left(D(T) / \operatorname{Ker} T, \sigma\left(D(T) / \operatorname{Ker} T, \operatorname{Ker} T^{\perp}\right)\right) \rightarrow\left(R(T), \sigma\left(F, F^{\prime}\right) \cap R(T)\right),
$$

i.e., $\check{T}^{-1}$ is weakly and therefore strongly continuous as the mapping

$$
\check{T}^{-1}:\left(R(T), \beta\left(F, F^{\prime}\right) \cap R(T)\right) \rightarrow\left(D(T) / \operatorname{Ker} T, \beta\left(D(T) / \operatorname{Ker} T, \operatorname{Ker} T^{\perp}\right)\right) .
$$

Taking into account the conditions a) and b) we obtain the validity of the following diagram:

$$
\begin{aligned}
& \left.\left(D(T) / \operatorname{Ker} T, \underset{\uparrow}{\beta}\left(E, E^{\prime}\right) \cap D(T) / \operatorname{Ker} T\right) \quad\left(R(T), \underset{\downarrow}{\beta\left(F, F^{\prime}\right.}\right) \cap R(T)\right) \\
& \left(D(T) / \operatorname{Ker} T, \beta\left(D(T) / \operatorname{Ker} T, \operatorname{Ker} T^{\perp}\right)\right) \leftarrow\left(R(T), \beta\left(R(T), F^{\prime} / R(T)^{\perp}\right)\right),
\end{aligned}
$$

where the arrows denote the continuous mappings. Hence we conclude that $T$ is strongly open.

Note that to prove the converse statement of Theorem 4, i.e., to prove that the above topologies coincide on $D(T) / \operatorname{Ker} T$ and $R(T)$, for the strongly and weakly open operator $T$ we should require, in addition to the a priori conditions of Theorem 4 , that $T$ be a weak homomorphism. Indeed, in that case, by virtue 
of the well known inequalities between strong topologies on $D(T) / \operatorname{Ker} T$ and $R(T)$ we obtain the validity of the following diagram:

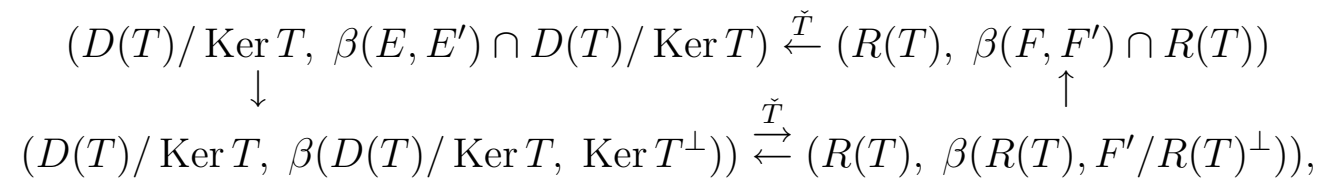

where the operators denoted by the arrows are continuous. Hence it follows that two identical operators in the diagram are isomorphisms, i.e., the above strong topologies on $D(T) / \operatorname{Ker} T$ and $R(T)$ coincide.

Let us now apply Theorem 2 to prove a sufficient condition for a weakly open operator to be open and strongly open.

Theorem 5. Let $T: E \rightarrow F$ be the weakly open linear operator of the LCS $\left(E, \mathfrak{T}_{1}\right)$ in the $\operatorname{LCS}\left(F, \mathfrak{T}_{2}\right)$. If the space $\left(R(T), \mathfrak{T}_{2} \cap R(T)\right)$ is a Mackey space, then $T$ is open. Moreover, if the graph of the operator $G(T)$ is barrelled, then the operator $T$ is strongly open.

Proof. For the linear operator $T$, let us consider the continuous operator $S$ : $G(T) \rightarrow F$ defined by the equality $S(e, T e)=T e, e \in D(T)$. According to Theorem 2.2 from [6] $T$ is weakly (resp. strongly) open if and only if $S$ is weakly (resp. strongly) open. Thus to prove our statement, it is sufficient to show that $S$ is a strong homomorphism. Indeed, we have the following natural decomposition of the operator $S=J \check{S} K$ and

$$
\begin{gathered}
\left(G(T) / \operatorname{Ker} S,\left(\mathfrak{T}_{1} \times \mathfrak{T}_{2}\right) / \operatorname{Ker} S\right) \stackrel{\check{S}}{\rightarrow}\left(R(T), \mathfrak{T}_{2} \cap R(T)\right) \stackrel{i d_{1}}{\rightleftarrows}\left(R(T), \tau\left(R(T), F^{\prime} / R(T)^{\perp}\right)\right) \\
\uparrow i d_{2} \\
\left(G(T) / \operatorname{Ker} S, \tau\left(G(T), G(T)^{\prime}\right) / \operatorname{Ker} S\right) \stackrel{i d_{3}}{\rightleftarrows}\left(G(T) / \operatorname{Ker} S, \tau\left(G(T) / \operatorname{Ker} S, \operatorname{Ker} S^{\perp}\right)\right),
\end{gathered}
$$

where each operator denoted by the arrows is continuous. Indeed, $i d_{1}$ is an isomorphism by virtue of the fact that $\mathfrak{T}_{2} \cap R(T)$ is the Mackey topology; $i d_{2}$ is continuous because the initial topology is weaker than the Mackey topology; $i d_{3}$ is an isomorphism because the quotient topology $\tau\left(G(T), G(T)^{\prime}\right) / \operatorname{Ker} S$ of the Mackey topology coincides with the Mackey topology $\tau\left(G(T) / \operatorname{Ker} S, \operatorname{Ker} S^{\perp}\right)$ on $G(T) / \operatorname{Ker} S ; \check{S}_{1}$ is an isomorphism because weak isomorphisms are also isomorphisms in the Makey topologies. This already implies that $S$ is a homomorphism of the space $\left(G(T),\left(\mathfrak{T}_{1} \times \mathfrak{T}_{2}\right) \cap G(T)\right)$ in $\left(F, \mathfrak{T}_{2}\right)$, i.e., $T$ is open.

Further, the barrelledness of the space $\left(G(T),\left(\mathfrak{T}_{1} \times \mathfrak{T}_{2}\right) \cap G(T)\right)$ means that the topologies $\left(\mathfrak{T}_{1} \times \mathfrak{T}_{2}\right) \cap G(T)$ and $\beta\left(G(T), G(T)^{\prime}\right)$ coincide on $G(T)$. Since $S$ is a homomorphism it follows that the spaces $\left(G(T) / \operatorname{Ker} S,\left(\mathfrak{T}_{1} \times \mathfrak{T}_{2}\right) / \operatorname{Ker} S\right)$ and $\left(R(T), \mathfrak{T}_{2} \cap R(T)\right)$ are barrelled and therefore $\left.\left(\mathfrak{T}_{1} \times \mathfrak{T}_{2}\right) / \operatorname{Ker} S\right)=\beta(G(T) /$ $\operatorname{Ker} S$, Ker $S^{\perp}$ ) and $\mathfrak{T}_{2} \cap R(T)=\beta\left(R(T), F^{\prime} / R(T)^{\perp}\right)$. This means that $T$ is strongly open.

This theorem shows that the conditions for the validity of the implication $e) \Longrightarrow d$ ) to be valid (Theorem 2.1 from [6]) can be weakened and made less cumbersome. 
We will now give conditions for a weak homomorphism to be also a homomorphism in the Mackey topologies.

Theorem 6. Let $A$ be a weak homomorphism of the LCS E in the LCS F. A is a $\mathfrak{T}_{k}$-homomorphism, i.e., a homomorphism of the space $\left(E, \tau\left(E, E^{\prime}\right)\right)$ in the space $\left(F, \tau\left(F, F^{\prime}\right)\right)$ if and only if $\tau\left(F, F^{\prime}\right) \cap A(E)=\tau\left(A(E), F^{\prime} / A(E)^{\perp}\right)$ on $A(E)$, where $\tau\left(F, F^{\prime}\right) \cap A(E)$ is the induced topology on $A(E)$, and $\tau\left(A(E), F^{\prime} / A(E)^{\perp}\right)$ is the Mackey topology on $A(E)$ with respect to the dual pair $\left\langle A(E), F^{\prime} \mid A(E)^{\perp}\right\rangle$.

The validity of this theorem follows from Theorem 1 with regard for the equality $\tau\left(E, E^{\prime}\right) / \operatorname{Ker} A=\tau\left(E / \operatorname{Ker} A, \operatorname{Ker} A^{\perp}\right)$ on $E / \operatorname{Ker} A$, which is obtained by Theorem 3 ([21, p. 277]).

Corollary 1. Let $A$ be a continuous weak homomorphism of the $L C S\left(E, \mathfrak{T}_{1}\right)$ in the $L C S\left(F, \mathfrak{T}_{2}\right)$ such that the space $\left(A(E), \mathfrak{T}_{2} \cap A(E)\right)$ is the Mackey space. Then $A$ is a homomorphism of the space $\left(E, \mathfrak{T}_{1}\right)$ in the space $\left(F, \mathfrak{T}_{2}\right)$.

This result generalizes the first part of Proposition 5 from [10, p.8].

Corollary 2. Let $A$ be a weak homomomorhism of the barrelled (resp. quasibarrelled) LCS $\left(E, \mathfrak{T}_{1}\right)$ in the $\operatorname{LCS}\left(F, \mathfrak{T}_{2}\right)$ such that the space $\left(A(E), \mathfrak{T}_{2} \cap\right.$ $A(E))$ is the Mackey space. Then $A$ is a strong homomorphism (resp. a $\mathfrak{T}_{b^{*}}$ homomorphism).

This result generalizes Proposition 21 from [2] as well Theorem 4.7.6 from $\left[11\right.$, p. 202], where it is not required of $\left(A(E), \mathfrak{T}_{2} \cap A(E)\right)$ to be the Mackey space.

Theorem 7. Let $A$ be a weak homomorphism of the LCS $\left(E, \mathfrak{T}_{1}\right)$ in the $\operatorname{LCS}\left(F, \mathfrak{T}_{2}\right) . A$ is a $\mathfrak{T}_{c}$-homomorphism, i.e., a homomorphism of the space $\left(E, \mathfrak{T}_{c}\left(E^{\prime}\right)\right)$ in the space $\left(F, \mathfrak{T}_{c}\left(F^{\prime}\right)\right)$ if and only if $\mathfrak{T}_{c}\left(E^{\prime}\right) / \operatorname{Ker} A=\mathfrak{T}_{c}\left(\operatorname{Ker} A^{\perp}\right)$ on $E / \operatorname{Ker} A$ and $\mathfrak{T}_{c}\left(F^{\prime}\right) \cap A(E)=\mathfrak{T}_{c}\left(F^{\prime} / A(E)^{\perp}\right)$ on $A(E)$.

We will now give examples of monomorphisms of the space of test functions $D$ and generalized functions $D^{\prime}$ on whose range the two topologies indicated in Theorem 7 are different. It is well known that the spaces $D$ and $D^{\prime}$ are connected with "the Pontryagin duality", i.e., the topology of each of these spaces coincides with a uniform convergence topology on strongly compact sets of the dual space. As shown in [22], this duality does not any longer extend to the quotient spaces of the spaces $D$ and $D^{\prime}$. This means that there exists a closed subspace $G$ of the space $D$ (resp. a closed subspace $M$ of the space $\left.D^{\prime}\right)$ such that the spaces $\left(G, \mathfrak{T}_{c}\left(D^{\prime}\right) \cap G\right)$ and $\left(G, \mathfrak{T}_{c}\left(D^{\prime} / G^{\perp}\right)\right)$ (resp. the spaces $\left(M, \mathfrak{T}_{c}(D) \cap M\right)$ and $\left(M, \mathfrak{T}_{c}\left(D / M^{\perp}\right)\right)$ are not isomorphic. By Theorem 7 this is equaivalent to that the monomorphism $J: G \rightarrow D$ (resp. the monomorphism $\left.J_{1}: M \rightarrow D^{\prime}\right)$ is not a $\mathfrak{T}_{c}$-monomorphism.

Consider this problem assuming that the spaces $E$ and $F$ are equipped with the so-called bornological topologies. Let $(E, \mathfrak{T})$ be a LCS. By $[21$, p. 380] there exists, on $E$, the strongest locally convex topology $\mathfrak{T}^{\times}$which possesses the same bounded sets as the topology $\mathfrak{T}$. For the topology $\mathfrak{T}^{\times}$the basis of 
neighborhoods of zero consists of all absolutely convex sets which absorb all bounded sets. $\left(E, \mathfrak{T}^{\times}\right)$is a bornological space and we call it the associated bornological space.

Theorem 8. Let $A$ be a weak homomorphism of the $L C S\left(E, \mathfrak{T}_{1}\right)$ in the $\operatorname{LCS}\left(F, \mathfrak{T}_{2}\right)$. A is a $\mathfrak{T}^{\times}$-homomorphism, i.e., a homomorphism of the space $\left(E, \mathfrak{T}_{1}^{\times}\right)$in $\left(F, \mathfrak{T}_{2}^{\times}\right)$if and only if $\mathfrak{T}_{1}^{\times} / \operatorname{Ker} A=\left(\mathfrak{T}_{1} / \operatorname{Ker} A\right)^{\times}$on $E / \operatorname{Ker} A$ and $\mathfrak{T}_{2}^{\times} \cap A(E)=\left(\mathfrak{T}_{2} \cap A(E)\right)^{\times}$on $A(E)$, where $\mathfrak{T}_{1}^{\times} /$Ker $A$ is the quotient topology of the topology $\mathfrak{T}_{1}^{\times},\left(\mathfrak{T}_{1} / \operatorname{Ker} A\right)^{\times}$is the associated bornological topology of the quotient topology $\mathfrak{T}_{1} / \operatorname{Ker} A, \mathfrak{T}_{2}^{\times} \cap A(E)$ is the induced topology on $A(E)$ of the topology $\mathfrak{T}_{2}^{\times}$and $\left(\mathfrak{T}_{2} \cap A(E)\right)^{\times}$is the associated bornological topology of the induced on $A(E)$ topology $\mathfrak{T}_{2} \cap A(E)$.

Proof. First we are to prove that each weakly continuous mapping $A$ is a continuous mapping of the space $\left(E, \mathfrak{T}_{1}^{\times}\right)$into $\left(F, \mathfrak{T}_{2}^{\times}\right)$. This follows from the local boundedness of the mapping $A$, i.e., $A$ transfroms the bounded subsets from $\left(E, \mathfrak{T}_{1}\right)$ into the same kind of subsets $\left(F, \mathfrak{T}_{2}\right)$. Therefore the following diagram is valid:

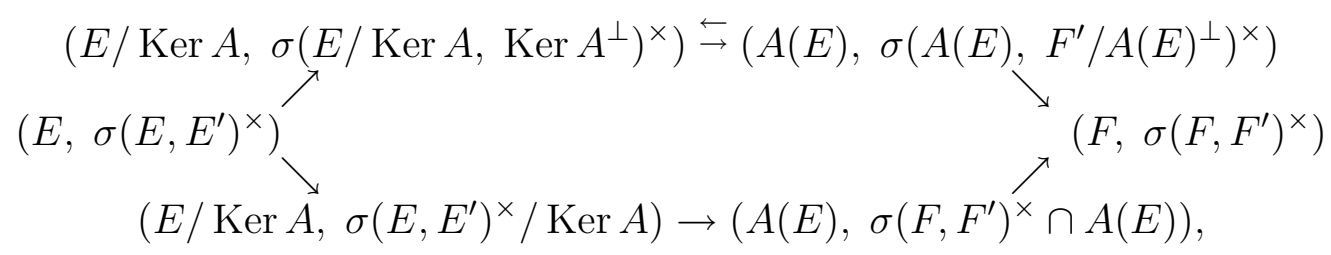

where the arrows denote the continuous mappings. Since the associated bornological topology depends only on the dual pair, the topologies $\sigma\left(E, E^{\prime}\right)$, $\sigma\left(E / \operatorname{Ker} A, \operatorname{Ker} A^{\perp}\right), \sigma\left(A(E), F^{\prime} / A(E)^{\perp}\right)$ and $\sigma\left(F, F^{\prime}\right)$ in the above diagram can be replaced by the topologies $\mathfrak{T}_{1}, \mathfrak{T}_{1} / \operatorname{Ker} A, \mathfrak{T}_{2} \cap A(E)$ and $\mathfrak{T}_{2}$, respectively.

Let us now prove the continuity of the identical mappings $(E / \operatorname{Ker} A$, $\left.\mathfrak{T}_{1}^{\times} / \operatorname{Ker} A\right) \rightarrow\left(E / \operatorname{Ker} A,\left(\mathfrak{T}_{1} / \operatorname{Ker} A\right)^{\times}\right)$and $\left(A(E),\left(\mathfrak{T}_{2} \cap A(E)\right)^{\times}\right) \rightarrow(A(E)$, $\left.\mathfrak{T}_{2}^{\times} \cap A(E)\right)$. Indeed, let $W$ be a $\left(\mathfrak{T}_{1} / \operatorname{Ker} A\right)^{\times}$-neighborhood. Then $W$ absorbs all $\mathfrak{T}_{1} / \operatorname{Ker} A$-bounded sets of the quotient space $E / \operatorname{Ker} A$. Therefore the set $K^{-1}(W)$ absorbs all $\mathfrak{T}_{1}$-bounded sets in $E$ and is a $\mathfrak{T}_{1}^{\times}$-neighborhood, where $K: E \rightarrow E / \operatorname{Ker} A$ is a canonical mapping. Hence $W=K\left(K^{-1} W\right)$ is a $\mathfrak{T}_{1}^{\times} / \operatorname{Ker} A$-neighborhood. Let now $U$ be a $\mathfrak{T}_{2}^{\times} \cap A(E)$-neighborhood in $A(E)$. Then $U=V \cap A(E)$, where $V$ is a $\mathfrak{T}_{2}^{\times}$-neighborhood and thus $V$ absorbs all bounded subsets in $\left(F, \mathfrak{T}_{2}\right)$. Hence it immediately follows that $U=V \cap A(E)$ absorbs all bounded sets $A(E)$, i.e., $U$ is a $\left(\mathfrak{T}_{2} \cap A(E)\right)^{\times}$-neighborhood in $A(E)$. By virtue of this reasoning the following diagram is valid:

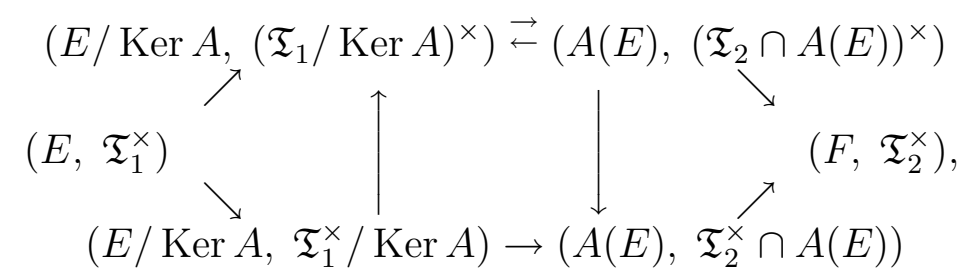


where the arrows denote the continuous mappings. The diagram readily provides the proof of our statement.

\section{On the Adjoint Operator to a Homomorhism Between Locally CONVEX SPACES}

It is well known that $A$ is a weak homomorphism of the LCS $E$ in the LCS $F$ with the closed range if and only if the adjoint mapping $A^{\prime}$ is a weak homomorphism with the weakly closed range $A^{\prime}\left(F^{\prime}\right)=\operatorname{Ker} A^{\perp}$. Then if $A=J \check{A} K$ is the decomposition of the weak homomorphism $A$ and $J^{\prime}, \check{A}^{\prime}$, and $K^{\prime}$ are the adjoints to the mappings $J, \check{A}$ and $K$, respectively, then $A^{\prime}=K^{\prime} \check{A}^{\prime} J^{\prime}$ is the natural decomposition of the weak homomorphism $A^{\prime}$. This means that $J^{\prime}$ is a canonical homomorphism of the space $\left(F^{\prime}, \sigma\left(F^{\prime}, F\right)\right)$ on $\left(A(E)^{\prime}, \sigma\left(A(E)^{\prime}, A(E)\right)\right)=$ $\left(F^{\prime} / \operatorname{Ker} A^{\prime}, \sigma\left(F^{\prime}, F\right) / \operatorname{Ker} A^{\prime}\right), K^{\prime}$ is a weak monomorphism of the space $\left((E / \operatorname{Ker} A)^{\prime}, \sigma\left((E / \operatorname{Ker} A)^{\prime}, E / \operatorname{Ker} A\right)\right)=\left(\operatorname{Ker} A^{\perp}, \sigma\left(E^{\prime} E\right) \cap \operatorname{Ker} A^{\perp}\right)=\left(A^{\prime}\left(F^{\prime}\right)\right.$, $\left.\sigma\left(E^{\prime}, E\right) \cap A^{\prime}\left(F^{\prime}\right)\right)$ in $\left(E^{\prime}, \sigma\left(E^{\prime}, E\right)\right)$, a $\check{A}^{\prime}$ is a weak isomorphism of the space $\left(F^{\prime} / \operatorname{Ker} A^{\prime}, \sigma\left(F^{\prime}, F\right) / \operatorname{Ker} A^{\prime}\right)$ on $\left(A^{\prime}\left(F^{\prime}\right), \sigma\left(E^{\prime}, E\right) \cap A^{\prime}\left(F^{\prime}\right)\right)$.

Let $\mathfrak{M}_{1}$ and $\mathfrak{M}_{2}$ be the saturated classes of bounded subsets in $E$ and $F$, respectively. Denote by $\mathfrak{T}_{\mathfrak{M}_{1}}(E)$ and $\mathfrak{T}_{\mathfrak{M}_{2}}(F)$ the topologies, on $E^{\prime}$ and $F^{\prime}$, of uniform convergence on the subsets from $\mathfrak{M}_{1}$ and $\mathfrak{M}_{2}$, respectively. Let $A$ be a weakly continuous mapping of the space $E$ into the space $F$; then the adjoint mapping $A^{\prime}$ is a continuous mapping of the space $\left(F^{\prime}, \mathfrak{T}_{\mathfrak{M}_{2}}(F)\right)$ in $\left(E^{\prime}, \mathfrak{T}_{\mathfrak{M}_{1}}(E)\right)$ if and only if $A\left(\mathfrak{M}_{1}\right) \subset \mathfrak{M}_{2}$. Hence it follows that the adjoint to the weak isomorphism of the space $E$ in the space $F$ is strong and a $\mathfrak{T}_{k^{-}}$ isomorphism. The example of the identical mapping $I$ of the normed space $(E,\|\|$.$) on \left(E, \sigma\left(E, E^{\prime}\right)\right)$ shows that its adjoint $I^{\prime}$ is a strong isomorphism, but $I$ is not an isomorphism.

Theorem 9. Let $E$ be $F$ locally convex spaces, $\mathfrak{M}_{1}$ and $\mathfrak{M}_{2}$ be the saturated classes of bounded sets in $E$ and $F$, respectively, and $A: E \rightarrow F$ be a weak homomorphism with the closed range $A(E)$. The adjoint mapping $A^{\prime}$ is a homomorphism of the space $\left(F^{\prime}, \mathfrak{T}_{\mathfrak{M}_{2}}(F)\right)$ in the space $\left(E^{\prime}, \mathfrak{T}_{\mathfrak{M}_{1}}(E)\right)$ if and only if:

a) $A\left(\mathfrak{M}_{1}\right) \subset \mathfrak{M}_{2}$ and $\overline{\mathfrak{M}}_{2}=\widetilde{\check{A}\left(\widehat{\mathfrak{M}}_{1}\right)}$, where $\overline{\mathfrak{M}}_{2}=\left\{M \in \mathfrak{M}_{2} ; M \subset A(E)\right\}=$ $\mathfrak{M}_{2} \cap A(E), \widehat{\mathfrak{M}}_{1}=K\left(\mathfrak{M}_{1}\right)=\left\{K(M) ; M \in \mathfrak{M}_{1}\right\}$, and $\widehat{\breve{A}\left(\widehat{\mathfrak{M}}_{1}\right)}$ is the saturated cover of the class $\check{A}\left(\widehat{\mathfrak{M}}_{1}\right)$;

b) $\mathfrak{T}_{\widehat{\mathfrak{M}}_{1}}(E / \operatorname{Ker} A)=\mathfrak{T}_{\mathfrak{M}_{1}}(E) \cap A^{\prime}\left(F^{\prime}\right)$ on $(E / \operatorname{Ker} A)^{\prime}=\operatorname{Ker} A^{\perp}=A^{\prime}\left(F^{\prime}\right)$, where $\mathfrak{T}_{\widehat{\mathfrak{M}}_{1}}$ is the topology of uniform convergence on the sets from $\widehat{\mathfrak{M}}_{1}$;

c) $\mathfrak{T}_{\mathfrak{M}_{2}}(F) / \operatorname{Ker} A^{\prime}=\mathfrak{T}_{\overline{\mathfrak{M}}_{2}}(A(E))$ on $A(E)^{\prime}=F^{\prime} / A(E)^{\perp}=F^{\prime} / \operatorname{Ker} A^{\prime}$.

The validity of this theorem follows from Theorem 1 if the latter is applied to the weak homomorphism $A^{\prime}$ of the space $\left(F^{\prime}, \sigma\left(F^{\prime}, F\right)\right)$ in the space $\left(E^{\prime}, \sigma\left(E^{\prime}, E\right)\right)$. One can also prove it in a straightforward manner analogously to the proof of Theorem 1 . 
Now we will give a few concrete reformulations of Theorem 9 for the most important topologies.

Theorem 10. Let $A$ be a weak homomorphism of the LCS E in the LCS $F$ with a weakly closed range. $A^{\prime}$ is a strong homomorphism, i.e., a homomorphism of the space $\left(F^{\prime}, \beta\left(F^{\prime}, F\right)\right)$ in the space $\left(E^{\prime}, \beta\left(E^{\prime}, E\right)\right)$ if and only if $\beta\left(F^{\prime}, F\right) / \operatorname{Ker} A^{\prime}=\beta\left(F^{\prime} / \operatorname{Ker} A^{\prime}, A(E)\right)$ on $F^{\prime} / \operatorname{Ker} A^{\prime}$ and $\beta\left(\operatorname{Ker} A^{\perp}, E / \operatorname{Ker} A\right)$ $=\beta\left(E^{\prime}, E\right) \cap \operatorname{Ker} A^{\perp}$ on $A^{\prime}\left(F^{\prime}\right)=\operatorname{Ker} A^{\perp}$.

Corollary 1. Let $A: E \rightarrow F$ be a homomorphism of the $(D F)$-space $(E, \mathfrak{T})$ in an arbitrary $\operatorname{LCS}(F, \mathfrak{T})$. Then $A^{\prime}$ is a strong homomorphism.

Note that Corollary 1 of Theorem 10 is valid for a space $\left(E, \mathfrak{T}_{1}\right)$ of type $(D F S)$, in particular, for the Vladimirov algebra [23, 24]. Moreover, if the space $\left(F, \mathfrak{T}_{2}\right)$ is also a space of type $(D F S)$, then the converse statement also holds true ([2, p. 105]), i.e., $A$ is a homomorphism if $A$ is continuous and $A^{\prime}$ is a strong homomorphism. In $\S 4$ Example 3 is given, showing that the latter statement is not valid for arbitrary $(D F)$-spaces.

Corollary 2. Let $A: E \rightarrow F$ be a weak homomorphism of the Fréchet space $E$ in the Fréchet space $F$. The adjoint operator $A^{\prime}$ is a strong homomorphism if and only if $\left.\beta\left((E / \operatorname{Ker} A)^{\prime}, E / \operatorname{Ker} A\right)\right)=\beta\left(E^{\prime}, E\right) \cap \operatorname{Ker} A^{\perp}$ on $(E / \operatorname{Ker} A)^{\prime}=$ $\operatorname{Ker} A^{\perp}$ and $\beta\left(A(E)^{\prime}, A(E)\right)=\beta\left(F^{\prime}, F\right) / \operatorname{Ker} A^{\prime}$ on $A(E)^{\prime}=F^{\prime} / \operatorname{Ker} A^{\prime}$.

From Theorem 12 [3] it follows that the Fréchet-Schwartz spaces $E$ and $F$ satisfy the conditions of Corollary 2. Moreover, similarly to the proof of Corollary 1 , one can prove that if $A$ is a homomorphism of the space $(F S)$ in an arbitrary LCS, then $A^{\prime}$ is a strong homomorphism. We will also indicate here yet another class of Fréchet spaces containing nonreflexive spaces satisfying the conditions of Corollary 2.

Proposition 11. Let $A$ be a homomorphism of the quojection $B \times \omega$ in the $\operatorname{LCS}\left(F, \mathfrak{T}_{2}\right)$, where $B$ is the Banach space and $\omega$ the space of all numerical sequences. Then the adjoint mapping $A^{\prime}$ is a strong homomorphism. Furthermore, if the space $\left(F, \mathfrak{T}_{2}\right)$ is a Fréchet space, the adjoint mapping of arbitrary order is a strong homomorphism.

Proof. Let us show that the conditions of Corollary 2 of Theorem 10 are fulfilled. Indeed, as is known, the quotient space of the space $B \times \omega$ is isomorphic either to the Banach space $B_{1}$ or to the space $B_{2} \times \omega$, where $B_{2}$ is also a Banach space. Therefore the space $\left.\left.((B \times \omega) / \operatorname{Ker} A)^{\prime}, \beta((B \times \omega) / \operatorname{Ker} A)^{\prime},(B \times \omega) / \operatorname{Ker} A\right)\right)$ is a strictly $(L B)$-space. Let us prove that the latter space is isomorphic to the space $\left(\operatorname{Ker} A^{\perp}, \beta\left(B^{\prime} \times \varphi, B \times \omega\right) \cap \operatorname{Ker} A^{\perp}\right)$, where $\varphi=\omega^{\prime}$ is the space of all finite sequences. Indeed, since $\operatorname{Ker} A$ is the quojection, we have the equality $\left((\operatorname{Ker} A)^{\prime}, \beta\left((\operatorname{Ker} A)^{\prime}, \operatorname{Ker} A\right)\right)=\left(\left(B^{\prime} \times \varphi\right) / \operatorname{Ker} A^{\perp}, \beta\left(B^{\prime} \times \varphi, B \times \omega\right) / \operatorname{Ker} A^{\perp}\right)$, where the latter is a strict $(L B)$-space. Then by virtue of the first part of Theorem 2 from [25] it turns out that the space $\left(\operatorname{Ker} A^{\perp}, \beta\left(B^{\prime} \times \varphi, B \times \omega\right) \cap\right.$ $\left.\operatorname{Ker} A^{\perp}\right)$ possesses the property $(\mathrm{T})$, i.e., it is also a strict $(L B)$-space. By virtue 
of the well-known theorem on the openness of a continuous mapping of strict $(L F)$-spaces from [2] we obtain the coincidence of the above-mentioned two topologies on Ker $A^{\perp}$.

By condition, the quotient space $(B \times \omega) / \operatorname{Ker} A$ is isomorphic to the space $\left(A(B \times \omega), \mathfrak{T}_{2} \cap A(B \times \omega)\right)$ and therefore the latter space is distinguished. Further, applying Theorem 8 from [3] we obtain the equality

$$
\left((A(B \times \omega))^{\prime}, \beta\left((A(B \times \omega))^{\prime}, A(B \times \omega)\right)\right)=\left(F^{\prime} / \operatorname{Ker} A^{\prime}, \beta\left(F^{\prime}, F\right) / \operatorname{Ker} A^{\prime}\right) .
$$

Therefore $A^{\prime}$ is a strong homomorphism. To prove that the second adjoint $A^{\prime \prime}$ to the homomorphism $A$, i.e., the adjoint to the mapping $A^{\prime}:\left(F^{\prime}, \beta\left(F^{\prime}, F\right)\right) \rightarrow$ $\left(E^{\prime}, \beta\left(E^{\prime}, E\right)\right)$ is a strong homomorphism of the space $\left(E^{\prime \prime}, \beta\left(E^{\prime \prime}, E^{\prime}\right)\right)$ in $\left(F^{\prime \prime}\right.$, $\beta\left(F^{\prime \prime}, F^{\prime}\right)$ ), we use Corollary 1 of Theorem 10 for the homomorphism $A^{\prime}$. The remaining part of our statement follows from the obvious fact that the strong bidual to the space $B \times \omega$ is identifiable with the space $B^{\prime \prime} \times \omega$.

It should be noted that, as follows from the proof of Proposition 11, each weakly closed subspace of the strongly dual space to the space $B \times \omega$ is a strict $(L B)$-space. By a similar reasoning one can prove that each closed subspace of the space $B \times \varphi$ is a strict $(L B)$-space.

It should also be noted that Proposition 11 does not hold for an arbitrary quojection. Indeed, in [3] an example is constructed of a canonical homomorphism of the Fréchet-Montel space on the Banach space, whose adjoint is not a strong homomorphism. It is indicated in [3] that, using this example, one can construct a canonical homomorphism $k$ of the quojection $\left(l^{p}\right)^{N}(1 \leq p<\infty)$ on its quotient space, such that the adjoint mapping $k^{\prime}$ will not be again a strong homomorphism. It is indicated furthermore that an analogous example of the canonical homomorphism can be constructed for the quojection $C$ ] 0,1 [ of the space of continuous functions on ] 0,1 [, which is equipped with a compact convergence topology. In these both cases the weakly closed subspace Ker $k^{\perp}$ in the strong topologies $\left.\beta\left(\operatorname{Ker} k^{\perp},\left(l^{p}\right)^{N} / \operatorname{Ker} k\right)\right)$ and $\beta\left(\operatorname{Ker} k^{\perp}, C\right] 0,1[/ \operatorname{Ker} k)$ is a strict $(L B)$-space, i.e., two of the three topologies given on Ker $k^{\perp}$ in [3] coincide, while in the third induced topology from the strongly dual $(L B)$-space, the subspace $\operatorname{Ker} k^{\perp}$ is not even a $(D F)$-space though it possesses a fundamental sequence of bounded sets. It should be specially noted that unlike the above-mentioned canonical homomorphism, the quotient spaces $\left(l^{p}\right)^{N} /$ Ker $k$ and $C] 0,1$ [ / Ker $k$ cannot be Banach ones by virtue of the following proposition.

Proposition 12. Let $k: E \rightarrow F$ be a canonical homomorphism of the prequojection $E$ on the Banach space $(F,\|\|$.$) . Then the adjoint mapping k^{\prime}$ is a strong homomorphism.

Proof. Indeed, the adjoint mapping $k^{\prime}$ is a strongly continuous and one-to-one mapping of the Banach space $\left(F^{\prime},\|.\|^{\prime}\right)$ in the strict $(L B)$-space $\left(E^{\prime}, \beta\left(E^{\prime}, E\right)\right)=$ $s . \lim _{\rightarrow} F_{n}$ with a weak and hence strongly closed image $k^{\prime}\left(F^{\prime}\right)=\operatorname{Ker} k^{\perp}$. By 
Theorem 4 from $[21$, p. 85$]$ we have that $k^{\prime}$ is a continuous mapping of the Banach space $F^{\prime}$ in some Banach space $F_{n_{\circ}}$, where $k^{\prime}\left(F^{\prime}\right)$ is closed.

Hence it follows that the strong topology on Ker $k^{\perp}$ coincides with the induced topology from $F_{n_{0}}$ and therefore from $\left(E^{\prime}, \beta\left(E^{\prime}, E\right)\right)$, i.e., $k^{\prime}$ is a strong monomorphism.

We do not know whether the quojection $E$ is isomorphic to the space $B \times \omega$ if the adjoint for an arbitrary homomorphism of the space $E$ in the LCS $F$ is a strong homomorphism.

We do not know either whether in Proposition 11 the space $\omega$ can be replaced by an arbitrary Fréchet-Schwartz space.

In [8] (see also [24, p. 105]) necessary and sufficient conditions were found in terms of the duality functor $D$ for:

a) the strong dual to the subspace to be identifiable with the quotient space of the strong dual and b) the strong dual to the quotient space to be identifiable with the subspace of the strong dual. Using these results in combination with Theorem 10 we obtain

Proposition 13. Let $A$ be a homomorphism of the LCS E in the LCS F. The adjoint mapping $A^{\prime}$ is a strong homomorphism if and only if $D_{M}^{1}(\operatorname{Ker} A)=$ 0 and $D_{M}^{+}(A(E))=0$, where $M$ is an arbitrary set of a sufficiently large cardinality, $D_{M}^{1}$ is the first derivative of the duality functor $D$, and $D_{M}^{+}$is an additional derivative.

It would be interesting to find similar relations (connections) for second adjoint mappings and other topologies to be considered below.

Theorem 14. Let $A$ be a weak homomorphism of the LCS E in the LCS F with a closed range. The adjoint mapping $A^{\prime}$ is a $\mathfrak{T}_{k}$-homomorphism, i.e., a homomorphism of the space $\left(F^{\prime}, \tau\left(F^{\prime}, F\right)\right)$ in $\left(E^{\prime}, \tau\left(E^{\prime}, E\right)\right)$ if and only if

$\left((E / \operatorname{Ker} A)^{\prime}, \tau\left((E / \operatorname{Ker} A)^{\prime}, E / \operatorname{Ker} A\right)\right)=\left(\operatorname{Ker} A^{\perp}, \tau\left(E^{\prime}, E\right) \cap \operatorname{Ker} A^{\perp}\right)$.

This theorem follows from Theorem 9 by virtue of the known equality $\left(A(E)^{\prime}\right.$, $\left.\tau\left(A(E)^{\prime}, A(E)\right)\right)=\left(F^{\prime} / \operatorname{Ker} A^{\prime}, \tau\left(F^{\prime}, F\right) / \operatorname{Ker} A^{\prime}\right)$, which is obtained from Theorem 4 ([21, p. 278]).

Theorem 15. Let $A$ be a weak homomorphism of the LCS E in the LCS F with a closed range. The conjugate mapping $A^{\prime}$ is a $\mathfrak{T}_{c}$-homomorphism, i.e., a homomorphism of the space $\left(F^{\prime}, \mathfrak{T}_{c}(F)\right)$ in the space $\left(E^{\prime}, \mathfrak{T}_{c}(E)\right)$ if and only if $\left((E / \operatorname{Ker} A)^{\prime}, \mathfrak{T}_{c}(E / \operatorname{Ker} A)\right)=\left(\operatorname{Ker} A^{\perp}, \mathfrak{T}_{c}(E) \cap \operatorname{Ker} A^{\perp}\right)$ and $\left(A(E)^{\prime}, \mathfrak{T}_{c}(A(E))\right.$ $=\left(F^{\prime} / \operatorname{Ker} A^{\prime}, \mathfrak{T}_{c}(F) / \operatorname{Ker} A^{\prime}\right)$.

Corollary 1. Let $A$ be a homomorphism of the Fréchet space $E$ in the quasicomplete LCS F. Then the adjoint mapping $A^{\prime}$ is a $\mathfrak{T}_{c}$-homomorphism.

This corollary follows from Theorems 5 and 6 ([21, p. 278]) and Theorem 15. 
Corollary 2. Let $A$ be a homomorphism of the Montel $(D F)$-space $E$ in the quasicomplete LCS F. Then the adjoint mapping $A^{\prime}$ is a $\mathfrak{T}_{c}$-homomorphism.

Corollary 3. Let $A$ be a monomorphism of the LCS E in the quasicomplete LCS F. Then the adjoint mapping $A^{\prime}$ is a $\mathfrak{T}_{c}$-homomorphism.

Let us consider now this problem assuming that the dual spaces are equipped with inductive topologies. The inductive topology $\mathfrak{T}_{I}(E)$ was introduced in $[15]$ as the strongest locally convex topology on $E^{\prime}$ preserving the boundedness of equicontinuous sets. This topology coincides with the strong topology, in particular for quasinormed spaces and differs from the latter topology in the case of nondistinguished Fréchet spaces.

Theorem 16. Let $A$ be a homomorphism of the LCS $\left(E, \mathfrak{T}_{1}\right)$ in the LCS $\left(F, \mathfrak{T}_{2}\right)$. The adjoint operator $A^{\prime}$ is a $\mathfrak{T}_{I}$-homomorphism, i.e., a homomorphism of the space $\left(F^{\prime}, \mathfrak{T}_{I}(F)\right)$ in $\left(E^{\prime}, \mathfrak{T}_{I}(E)\right)$ if and only if $\left((E / \operatorname{Ker} A)^{\prime}, \mathfrak{T}_{I}(E / \operatorname{Ker} A)\right)=$ $\left(\operatorname{Ker} A^{\perp}, \mathfrak{T}_{I}(E) \cap \operatorname{Ker} A^{\perp}\right)$.

In [7] (see also [8]) sufficient conditions were obtained for the adjoint to the homomorphism to be a $\mathfrak{T}_{I}$-homomorphism. From these results and Theorem 16 it follows that if for the homomorphism $A$ the kernel $\operatorname{Ker} A$ is a quasinormed metrizable LCS, then $\left((E / \operatorname{Ker} A)^{\prime}, \mathfrak{T}_{I}(E / \operatorname{Ker} A)\right)=\left(\operatorname{Ker} A^{\perp}, \mathfrak{T}_{I}(E) \cap \operatorname{Ker} A^{\perp}\right)$ and therefore $A^{\prime}$ is a $\mathfrak{T}_{I}$-homomorphism.

\section{On the Second Adjoint Mapping to a Homomorphism Between Locally Convex Spaces}

Let $(E, \mathfrak{T})$ be a LCS, $E^{\prime}$ its dual space and $E^{\prime \prime}$ its bidual space, i.e., $E^{\prime \prime}=$ $\left(E^{\prime}, \beta\left(E^{\prime}, E\right)\right)^{\prime}$. For the weakly continuous linear mapping $A$ of the LCS $\left(E, \mathfrak{T}_{1}\right)$ in the LCS $\left(F, \mathfrak{T}_{2}\right)$ we define the second adjoint mapping $A^{\prime \prime}$ on $E^{\prime \prime}$ by means of the equality

$$
\left\langle A^{\prime \prime} x^{\prime \prime}, y^{\prime}\right\rangle=\left\langle x^{\prime}, A^{\prime} y^{\prime}\right\rangle,
$$

assuming that it is valid for all $x^{\prime \prime} \in E^{\prime \prime}$ and $y^{\prime} \in F^{\prime}$. It is obvious that the restriction of $A^{\prime \prime}$ on $E$ coincides with $A$. Since $A^{\prime \prime}$ is a continuous mapping of the space $\left(E^{\prime \prime}, \sigma\left(E^{\prime \prime}, E^{\prime}\right)\right)$ into the space $\left(F^{\prime \prime}, \sigma\left(F^{\prime \prime}, F^{\prime}\right)\right)$ and $E \sigma\left(E^{\prime \prime}, E^{\prime}\right)$-dense in $E^{\prime \prime}$, we see that $A^{\prime \prime}$ is the continuation of $A$ on $E^{\prime \prime}$. By $\sigma\left(E^{\prime \prime}, E^{\prime}\right)$ (resp. $\left.\beta\left(E^{\prime \prime}, E^{\prime}\right)\right)$ we denote a weak (resp. strong) topology of the dual pair $\left\langle E^{\prime}, E^{\prime \prime}\right\rangle$ on $E^{\prime \prime}$, while by $\mathfrak{T}_{n}\left(E^{\prime}\right)$ we denote a natural topology on $E^{\prime \prime}$, i.e., a topology of uniform convergence on equicontinuous sets of the dual space $E^{\prime}$.

In this section we will deal with the second adjoint mapping to the homomorphism in the above-mentioned topologies of the second dual spaces. Conditions will be derived under which the second adjoint is again a homomorphism irrespective of whether the adjoint mapping is a strong homomorphism. First we will give simple statements on the double adjoint to a weak homomorhism which are formulated on the basis of the well known results and needed for our further discussion. 
Proposition 17. Let $A$ be a weakly continuous mapping of the LCS $\left(E, \mathfrak{T}_{1}\right)$ in the $\operatorname{LCS}\left(F, \mathfrak{T}_{2}\right)$. Then the following statements are valid:

a) The second adjoint mapping $A^{\prime \prime}$ is a weak homomorphism, i.e., a homomorphism of the space $\left(E^{\prime \prime}, \sigma\left(E^{\prime \prime}, E^{\prime}\right)\right)$ in the space $\left(F^{\prime \prime}, \sigma\left(F^{\prime \prime}, F^{\prime}\right)\right)$ if and only if the range $A^{\prime}\left(F^{\prime}\right)$ is $\sigma\left(E^{\prime}, E^{\prime \prime}\right)$-closed in $\left(E^{\prime}, \sigma\left(E^{\prime}, E^{\prime \prime}\right)\right)$, where $\sigma\left(E^{\prime}, E^{\prime \prime}\right)$ is a weak topology of the dual pair $\left\langle E^{\prime}, E^{\prime \prime}\right\rangle$ on $E^{\prime}$;

b) If $A$ is a weak homomorphism of the space $E$ in the space $F$, then $A^{\prime \prime}$ is a weak homomorphism of the space $\left(E^{\prime \prime}, \sigma\left(E^{\prime \prime}, E^{\prime}\right)\right)$ in the space $\left(F^{\prime \prime}, \sigma\left(F^{\prime \prime}, F^{\prime}\right)\right)$. Further, there is an example of a weakly continuous mapping which is not weakly open, while the second adjoint mapping is a weak homomorphism.

c) The range $A^{\prime \prime}\left(E^{\prime \prime}\right)$ is $\sigma\left(F^{\prime \prime}, F^{\prime}\right)$-closed if and only if $A^{\prime}$ is a weak homomorphism of the space $\left(F^{\prime}, \sigma\left(F^{\prime}, F^{\prime \prime}\right)\right)$ in the space $\left(E^{\prime}, \sigma\left(E^{\prime}, E^{\prime \prime}\right)\right)$.

d) If $A$ is a weak homomorphism of the space $E$ in the space $F$ whose adjoint mapping is a strong homomorphism, then $A^{\prime \prime}$ is a weak homomorphism of the space $\left(E^{\prime \prime}, \sigma\left(E^{\prime \prime}, E^{\prime}\right)\right)$ in the space $\left(F^{\prime \prime}, \sigma\left(F^{\prime \prime}, F^{\prime}\right)\right)$ with a closed range. Moreover, the following equalities are valid: $A(E)^{\prime \prime}=\left(\operatorname{Ker} A^{\prime}\right)^{\perp}=A(E)^{\perp \perp}=A^{\prime \prime}\left(E^{\prime \prime}\right)$ and $(E / \operatorname{Ker} A)^{\prime \prime}=\left(\operatorname{Ker} A^{\perp}\right)^{\prime}=E^{\prime \prime} / \operatorname{Ker} A^{\prime \prime}=\left(A^{\prime}\left(F^{\prime}\right)\right)^{\prime}$.

Theorem 18. Let $A$ be a weak homomorphism of the LCS E in the LCS F with a closed range whose adjoint mapping $A^{\prime}$ is a strong homomorhism. Then the following statements are equivalent:

a) The second adjoint mapping $A^{\prime \prime}$ is a strong homomorphism of the space $\left(E^{\prime \prime}, \beta\left(E^{\prime \prime}, E^{\prime}\right)\right)$ in the space $\left(F^{\prime \prime}, \beta\left(\left(F^{\prime \prime}, F^{\prime}\right)\right)\right.$.

b) We have the equalities $\beta\left(\left(A^{\prime}\left(F^{\prime}\right)^{\prime}, A^{\prime}\left(F^{\prime}\right)\right)=\beta\left(E^{\prime \prime}, E^{\prime}\right) / \operatorname{Ker} A^{\prime \prime}\right.$ on $\left(A^{\prime}\left(F^{\prime}\right)^{\prime}\right.$ $=E^{\prime \prime} / \operatorname{Ker} A^{\prime \prime}$ and $\beta\left(\left(F^{\prime} / \operatorname{Ker} A^{\prime}\right)^{\prime}, F^{\prime} / \operatorname{Ker} A^{\prime}\right)=\beta\left(F^{\prime \prime}, F^{\prime}\right) \cap A^{\prime \prime}\left(F^{\prime \prime}\right)$ on $\left(E^{\prime} /\right.$ Ker $\left.A^{\prime}\right)^{\prime}=A^{\prime \prime}\left(F^{\prime \prime}\right)$, where $A^{\prime}\left(F^{\prime}\right)$ is considered in the induced topology $\beta\left(E^{\prime}, E\right) \cap$ $A^{\prime}\left(F^{\prime}\right)$, and the quotient space $F^{\prime} / \operatorname{Ker} A^{\prime}$ in the quotient topology.

c) We have the equalities $\beta\left((E / \operatorname{Ker} A)^{\prime \prime},(E / \operatorname{Ker} A)^{\prime}\right)=\beta\left(E^{\prime \prime}, E^{\prime}\right) / \operatorname{Ker} A^{\prime \prime}$ on $(E / \operatorname{Ker} A)^{\prime \prime}=E^{\prime \prime} / \operatorname{Ker} A^{\prime \prime}$ and $\beta\left(A(E)^{\prime \prime}, A(E)^{\prime}\right)=\beta\left(F^{\prime \prime}, F^{\prime}\right) \cap A^{\prime \prime}\left(F^{\prime \prime}\right)$ on $A(E)^{\prime \prime}=A^{\prime \prime}\left(E^{\prime \prime}\right)$, where $\beta\left((E / \operatorname{Ker} A)^{\prime \prime},(E / \operatorname{Ker} A)^{\prime}\right)$ is a strong topology of the space $(E / \operatorname{Ker} A)^{\prime \prime}$, and $\beta\left(A(E)^{\prime \prime}, A(E)^{\prime}\right)$ is a strong topology of the space $A(E)^{\prime \prime}$.

The following diagram is helpful in investigating the second adjoint to the homomorphism:

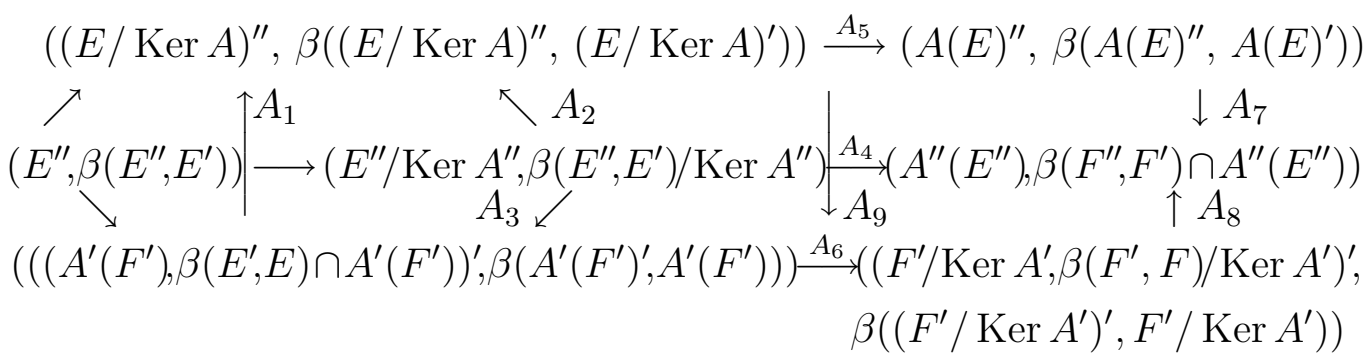

where $A_{1}, \ldots, A_{9}$ denote the continuous identical mappings. In particular, in the a priori conditions of Theorem 18 one can say that all mappings $A_{1}-A_{9}$ are continuous algebraic isomorphisms, and the mappings $A_{1}, A_{5}, A_{6}$ and $A_{9}$ are topological isomorphisms, while Theorem 18 states that $A_{4}$ is a topological 
isomorphism if and only if the operators $A_{3}$ and $A_{8}$ and, accordingly, $A_{2}$ and $A_{7}$ are topological isomorphisms. Hence in particular it follows that $A_{4}$ cannot be a topological isomorphism when $A_{5}$ and $A_{6}$ are topological isomorphisms.

Corollary 1. Let $k$ be a homomorphism of the $(D F)$-space $E$ on the $(D F)$ space $F$ such that $\left(k^{\prime}\left(F^{\prime}\right), \beta\left(E^{\prime}, E\right) \cap k^{\prime}\left(F^{\prime}\right)\right)$ is a distinguished Fréchet space. Then $k^{\prime \prime}$ is a strong homomorphism $\left(E^{\prime \prime}, \beta\left(E^{\prime \prime}, E^{\prime}\right)\right)$ on $\left(F^{\prime \prime}, \beta\left(F^{\prime \prime}, F^{\prime}\right)\right)$.

Corollary 2. Let $A$ be a homomorphism of the Fréchet space $E$ in the Fréchet space $F$ such that $A^{\prime}$ is a strong homomorphism. Then $A^{\prime \prime}$ is a strong homomorphism.

The validity of this statement follows from the fact that all spaces in the above diagram are Fréchet spaces. We do not know whether Corollary 2 holds without the requirement that $A^{\prime}$ be strongly homomorphic.

A linear operator $A:\left(E, \mathfrak{T}_{1}\right) \rightarrow\left(F, \mathfrak{T}_{2}\right)$ is called a nearly open mapping of the LCS $\left(E, \mathfrak{T}_{1}\right)$ into the $\operatorname{LCS}\left(F, \mathfrak{T}_{2}\right)$ if the $\mathfrak{T}_{2}$-closure of the image $\overline{A(U)}$ of each $\mathfrak{T}_{1}$-neighborhood $U$ is a $\mathfrak{T}_{2}$-neighborhood in $\overline{A(E)}$.

Theorem 19. Let $A$ be a continuous and nearly open operator of the LCS $\left(E, \mathfrak{T}_{1}\right)$ in the $\operatorname{LCS}\left(F, \mathfrak{T}_{2}\right)$. Then the second adjoint mapping $A^{\prime \prime}$ is nearly weakly open in natural topologies, i.e., the $\sigma\left(F^{\prime \prime}, F^{\prime}\right)$-closure of the $A^{\prime \prime}$-image of each $\mathfrak{T}_{n}\left(E^{\prime}\right)$-neighborhood of the space $E^{\prime \prime}$ is a $\mathfrak{T}_{n}\left(F^{\prime}\right)$-neighborhood in $F^{\prime \prime}$.

It should be noted that if in the conditions of Theorem $19 A$ was even a homomorphism ( $A$ is such when $E$ is a Pták space, i.e., B-complete), then, one cannot claim more than in Theorem 19. We will however give cases in which this is possible.

Corollary 1. Let $A$ be a continuous and nearly open mapping of the LCS $\left(E, \mathfrak{T}_{1}\right)$ into the $\operatorname{LCS}\left(F, \mathfrak{T}_{2}\right)$. Assume, further, that for any $\mathfrak{T}_{1}$-neighborhood

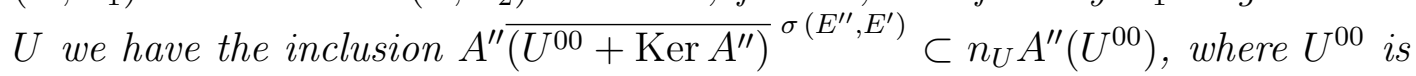
the bipolar of the set $U$ in $E^{\prime \prime}$ and $n_{U} \in N$. Then $A^{\prime \prime}$ is a homomorphism in natural topologies, i.e., $A^{\prime \prime}$ is a homomorphism of the space $\left(E^{\prime \prime}, \mathfrak{T}_{n}\left(E^{\prime}\right)\right)$ in the space $\left(F^{\prime \prime}, \mathfrak{T}_{n}\left(F^{\prime}\right)\right)$.

Corollary 2. Let $A$ be a continuous and nearly open mapping of the LCS $\left(E, \mathfrak{T}_{1}\right)$ into the LCS $\left(F, \mathfrak{T}_{2}\right)$ and the natural topology $\mathfrak{T}_{n}\left(E^{\prime}\right)$ of the space $E^{\prime \prime}$ be compatible with the duality $\left\langle E^{\prime \prime}, E^{\prime}\right\rangle$. Then $A^{\prime \prime}$ is a homomorphism in natural topologies.

This result generalizes Theorem 4 from [10], p. 11]. Note that if the spaces $\left(E, \mathfrak{T}_{1}\right)$ and $\left(F, \mathfrak{T}_{2}\right)$ are quasibarrelled, then natural topologies coincide with strong topologies on $E^{\prime \prime}$ and $F^{\prime \prime}$. Therefore in that case we can speak of strong homomorphisms in Corollaries 1 and 2. 
Proposition 20. Let $A$ be a homomorphism of the LCS $\left(E, \mathfrak{T}_{1}\right)$ in the LCS $\left(F, \mathfrak{T}_{2}\right)$ such that $(E / \operatorname{Ker} A)^{\prime \prime}=E^{\prime \prime} / \operatorname{Ker} A^{\prime \prime}$. The second adjoint mapping $A^{\prime \prime}$ is a homomorphism in natural topologies if and only if $\left((E / \operatorname{Ker} A)^{\prime \prime}, \mathfrak{T}_{n}\left(\operatorname{Ker} A^{\perp}\right)\right)$ $=\left(E^{\prime \prime} / \operatorname{Ker} A^{\prime \prime}, \mathfrak{T}_{n}\left(E^{\prime}\right) / \operatorname{Ker} A^{\prime \prime}\right)$.

There is an example of the Fréchet space $E$ and the canonical homomorphism $k: E \rightarrow E / \operatorname{Ker} k$ for which $(E / \operatorname{Ker} k)^{\prime \prime} \supset E^{\prime \prime} / \operatorname{Ker} k^{\prime \prime}$ and this inclusion is the proper one. Indeed, let $E=\left(l^{1}\right)^{N}$ and $F$ be a closed subspace of the space $E$ such that on $F^{\perp}$ two strong topologies give different dual spaces [3]. In that case if $k: E \rightarrow E / F$, then

$$
(k(E))^{\prime \prime}=(E / F)^{\prime \prime}=\left((E / F)^{\prime}, \beta\left((E / F)^{\prime}, E / F\right)\right)^{\prime} \nsupseteq\left(F^{\perp}, \beta\left(E^{\prime}, E\right) \cap F^{\perp}\right)^{\prime}=E^{\prime \prime} / \operatorname{Ker} k^{\prime \prime} .
$$

We do not know whether the second adjoint mapping $k^{\prime \prime}$ in this example is a strong homomorphism.

\section{Remarks on Some Homomorphisms of Fréchet Spaces, $(D F)$-Spaces, Strict $(L F)$-Spaces And Their Adjoint Mappings}

Example 1. We know of the well known rather difficult example of the Fréchet-Montel space $(E, \mathfrak{T})$ from [3] (see also [10, p. 22]) whose quotient topology is isomorphic to the Banach space $l^{1}$. Let $k: E \rightarrow l^{1}$ be this canonical homomorphism, i.e., the quotient space $\mathfrak{T} /$ Ker $k$ coincides with the topology of the norm of the space $l^{1}$. We know that $k$ is a strong homomorphism, i.e., $\beta\left(E, E^{\prime}\right) / \operatorname{Ker} k=\beta\left(l^{1}, l^{\infty}\right)$ on $l^{1}$. By virtue of the results from $\S 1 k$ is also a $\mathfrak{T}_{b^{*-}}$ homomorphism (the Corollary of Theorem 3), a $\mathfrak{T}_{k}$-homomorphism (Corollary 1 of Theorem 6) and a $\mathfrak{T}^{\times}$-homomorphism. However $k$ is not a $\mathfrak{T}_{c^{-}}$-homomorphism since $\mathfrak{T}_{c}\left(E^{\prime}\right) / \operatorname{Ker} k=\beta\left(E, E^{\prime}\right) / \operatorname{Ker} k=\beta\left(l^{1}, l^{\infty}\right) \neq \mathfrak{T}_{c}\left(l^{\infty}\right)$. As is known [3], the adjoint mapping $k^{\prime}$ is not a strong monomorphism and therefore

$$
\left((E / \operatorname{Ker} k)^{\prime}, \beta\left((E / \operatorname{Ker} k)^{\prime}, E / \operatorname{Ker} k\right)\right)=\left(l^{\infty}, \beta\left(l^{\infty}, l^{1}\right)\right) \neq\left(k^{\prime}\left(l^{\infty}\right), \beta\left(E^{\prime}, E\right) \cap k^{\prime}\left(l^{\infty}\right)\right) .
$$

Further, as is known, the topology $\beta\left(E^{\prime}, E\right)=\mathfrak{T}_{c}\left(E^{\prime}\right)$ induces, on $k^{\prime}\left(l^{\infty}\right)=$ Ker $k^{\perp}$, a uniform convergence topology on all relatively compact subsets $E$ / Ker $k=l^{1}$. Hence we also find that $\left(l^{\infty}, \mathfrak{T}_{c}\left(l^{1}\right)\right)=\left(k^{\prime}\left(l^{\infty}\right), \mathfrak{T}_{c}(E) \cap k^{\prime}\left(l^{\infty}\right)\right)$, i.e., $k^{\prime}$ is a $\mathfrak{T}_{c}$-monomorphism by virtue of Corollary 1 of Theorem 15 . From Theorem 16 it follows that $k^{\prime}$ is not a $\mathfrak{T}_{I}$-monomorphism since in the topo$\operatorname{logy} \mathfrak{T}_{I}(E) \cap k^{\prime}\left(l^{\infty}\right)=\mathfrak{T}_{c}(E) \cap k^{\prime}\left(l^{\infty}\right)$ the space $k^{\prime}\left(l^{\infty}\right)$ is not a bornological space, while $\left((E / \operatorname{Ker} k)^{\prime}, \mathfrak{T}_{I}(E / \operatorname{Ker} k)\right)=l^{\infty} . k^{\prime}$ is a monomorphism of the space $\left(l^{\infty}, \sigma\left(l^{\infty}, l^{1}\right)\right)$ in the space $\left(E^{\prime}, \sigma\left(E^{\prime}, E\right)\right)$ with the $\sigma\left(E^{\prime}, E\right)$-closed image Ker $k^{\perp}$, but $k^{\prime}$ is not any longer a monomorphism of the space $\left(l^{\infty}, \sigma\left(l^{\infty},\left(l^{\infty}\right)^{\prime}\right)\right)$ in the space $\left(E^{\prime}, \sigma\left(E^{\prime}, E\right)\right)$ since $\sigma\left(l^{\infty}, l^{1}\right)<\sigma\left(l^{\infty},\left(l^{\infty}\right)^{\prime}\right)$. Therefore, though $k^{\prime \prime}$ is a weak homomorphism of the space $\left(E^{\prime \prime}, \sigma\left(E^{\prime \prime}, E^{\prime}\right)\right)=\left(E, \sigma\left(E, E^{\prime}\right)\right)$ in $\left.\left(\left(l^{\infty}\right)^{\prime}, \sigma\left(\left(l^{\infty}\right)^{\prime}, l^{\infty}\right)\right)\right)$ on account of the statement b) of Proposition 17, but it does not any longer have the $\sigma\left(\left(l^{\infty}\right)^{\prime}, l^{\infty}\right)$-closed image in $\left(l^{\infty}\right)^{\prime}$. From Corollary 2 of Theorem 19 we also conclude that $k^{\prime \prime}$ is a strong homomorphism of the Fréchet space $E^{\prime \prime}=E$ in $\left(\left(l^{\infty}\right)^{\prime}, \beta\left(\left(l^{\infty}\right)^{\prime}, l^{\infty}\right)\right)=\left(l^{\infty}\right)^{\prime}$ with a strongly closed and weakly dense image. 
Example 2. Let us consider a bornological $(D F)$-space $(F, \mathfrak{T})$. By virtue of Theorem 5 ([21, p. 403]) it can be represented in the form of an inductive limit of an increasing sequence of normed spaces $\left\{\left(F_{n},\|\cdot\|_{n}\right)\right\}$ with respect to the mapping $I_{n m}:\left(F_{n},\|\cdot\|_{n}\right) \rightarrow\left(F_{m},\|\cdot\|_{m}\right)(n \leq m)$. Then the space $(F, \mathfrak{T})$ is isomorphic to the quotient space $\bigoplus_{n \in N} F_{n} / H$ of the sum $\bigoplus_{n \in N} F_{n}$ with respect to the subspace $H$ spanned on elements of the form $x-I_{n m}(x)$, where $x \in E_{n} \quad(n \in N)$. Let $k: E=\bigoplus_{n \in N} F_{n} \rightarrow E / H=F$ is a canonical homomorphism. Then $k$ is a $\mathfrak{T}_{b^{*-}}$ homomorphism (the Corollary of Theorem 3 ). Further, if the space $(F, \mathfrak{T})$ is barrelled, then $k$ is a strong homomorphism (the Corollary of Theorem 2 ). $k$ is also a $\mathfrak{T}_{k}$-homomorphism (Corollary 1 of Theorem 6 ) since the bornological space is a Mackey space by virtue of Theorem 1 ([21, p. 379]). The operator $k$ is also a $\mathfrak{T}^{\times}$-homomorphism.

The adjoint mapping $k^{\prime}$ is a strong monomorphism by virtue of Corollary 1 of Theorem 10 . Using the results of $\S 2$ one can prove that $k^{\prime}$ is also a monomorphism in some other topologies of dual spaces. In particular, $k^{\prime}$ is a $\mathfrak{T}_{k}$-monomorphism and a $\mathfrak{T}_{I^{-}}$-monomorphism. The second adjoint mapping $k^{\prime \prime}$ is a strong homomorphism if and only if the space $\left(H^{\perp}, \beta\left(E^{\prime}, E\right) \cap H^{\perp}\right)=$ $\left((E / H)^{\prime}, \beta\left((E / H)^{\prime},(E / H)\right)\right)$ is a distinguished Fréchet space. The example given in [26] shows that the space $\left(H^{\perp}, \beta\left((E / H)^{\prime}, E / H\right)\right)$ is not always distinguished. Hence there exists an example of a canonical homomorphism of the bornological $(D F)$-space whose double adjoint is not any longer a strong homomorphism though its first adjoint is a strong homomorphism. By virtue of Proposition 14 from [3] we also see that if $(F, \mathfrak{T})$ is the bornological $(D F)$ space satisfying the strict Mackey condition, then the mapping $k^{\prime \prime}$ is a strong homomorphism and its arbitrary adjoint mapping is also such. The same result takes place if $(F, \mathfrak{T})$ is a strict $(L B)$-space.

Example 3. Let $(F, \mathfrak{T})$ be a nonbornological $(D F)$-space and $F_{1}=\left(F, \mathfrak{T}^{\times}\right)$, where $\mathfrak{T}^{\times}$is the associative bornological topology $((F, \mathfrak{T})$ can be quasibarrelled $[27])$. By virtue of Example 2 we have $F_{1}=\left(F, \mathfrak{T}^{\times}\right)=\left(E / \operatorname{Ker} k_{1}, \mathfrak{T}_{1} / \operatorname{Ker} k_{1}\right)$, where $\left(E, \mathfrak{T}_{1}\right)$ is the sum of normed spaces and $k_{1}: E \rightarrow\left(E / \operatorname{Ker} k_{1}, \mathfrak{T}_{1} / \operatorname{Ker} k_{1}\right)$ $=F_{1}$ is a canonical mapping. It is obvious that $k_{1}^{\prime}$ is a strong monomorphism with a weakly closed image $k_{1}^{\prime}\left(F_{1}^{\prime}\right)=\operatorname{Ker} k_{1}^{\perp}$. Let also define the mapping $k_{2}: E \rightarrow F$ by the equality $k_{2} x=k_{1} x$ for all $x \in E$. The mapping $k_{2}$ is a continuous mapping of the bornological $(D F)$-space $\left(E, \mathfrak{T}_{1}\right)$ into the $(D F)$-space $(F, \mathfrak{T})$ which is not open. The mapping $k_{2}$ will not be a weak homomorphism if the space $(F, \mathfrak{T})$ is not a Mackey space since in that case it would be a homomorphism by virtue of Corollary 1 of Theorem 6 .

Let $I:\left(F, \mathfrak{T}^{\times}\right) \rightarrow(F, \mathfrak{T})$ be the identical mapping. Then $k_{2}=I \circ k_{1}$. Further, $k_{2}^{\prime}=k_{1}^{\prime} \circ I^{\prime}$ is a monomorphism of the space $\left(F^{\prime}, \sigma\left(F^{\prime}, F\right)\right)$ in $\left(E^{\prime}, \sigma\left(E^{\prime}, E\right)\right)$, but $k_{2}^{\prime}\left(F^{\prime}\right)$ is not $\sigma\left(E^{\prime}, E\right)$-closed in $E^{\prime}$ and therefore does not coincide with its $\sigma\left(E^{\prime}, E\right)$-closure $\operatorname{Ker} k_{2}^{\perp}=\operatorname{Ker} k_{1}^{\perp}=k_{1}^{\prime}\left(F_{1}^{\prime}\right)$ in $E^{\prime}$. Let us prove that if $k_{2}^{\prime}\left(F^{\prime}\right) \nsubseteq k_{1}^{\prime}\left(F_{1}^{\prime}\right)$ ([3], see also [21, p. 388]), then $k_{2}^{\prime}\left(F^{\prime}\right)$ is strongly closed in 
$k_{1}^{\prime}\left(F_{1}^{\prime}\right)$, i.e., $\left(F^{\prime}, \beta\left(F^{\prime}, F\right)\right)$ is closed in $\left(F_{1}^{\prime}, \beta\left(F_{1}^{\prime}, F_{1}\right)\right)$ and is a closed subspace of the space $\left(E^{\prime}, \beta\left(E^{\prime}, E\right)\right)$. Indeed, let $\left\{B_{n}\right\}$ be a fundamental sequence of bounded sets in $(F, \mathfrak{T})$ and $B_{n, 1}=I^{-1}\left(B_{n}\right)$, i.e., $B_{n}=I\left(B_{n, 1}\right)$. It is obvious that $\left\{B_{n, 1}\right\}$ is again a fundamental sequence in $\left(F, \mathfrak{T}^{\times}\right)$. By taking polars on both sides in the above equality in $F^{\prime}$ we obtain $B_{n}^{\circ}=I\left(B_{n, 1}\right)^{\circ}=I^{\prime(-1)}\left(B_{n, 1}^{\circ}\right)$, i.e., $I^{\prime}\left(B_{n}^{\circ}\right)=B_{n, 1}^{\circ} \cap I^{\prime}\left(F^{\prime}\right)$. This means that $\beta\left(F_{1}^{\prime}, F_{1}\right)$ induces on $F^{\prime}$ the topology $\beta\left(F^{\prime}, F\right)$ and therefore $F^{\prime}$ is closed in $\left(F_{1}^{\prime}, \beta\left(F_{1}^{\prime}, F_{1}\right)\right)$, i.e., $I^{\prime}$ is a strong monomorphism with a strongly closed image in $F_{1}^{\prime}$. Therefore the mapping $I$ is an example of a continuous mapping of the $(D F)$-space into the same space whose adjoint is a strong monomorphism.

Example 4. It is well known that the Fréchet space $\left(E, \mathfrak{T}_{1}\right)$ is isomorphic to the closed subspace of the product of Banach spaces $\left(F, \mathfrak{T}_{2}\right)$. Let $J:\left(E, \mathfrak{T}_{1}\right) \rightarrow$ $\left(F, \mathfrak{T}_{2}\right)$ be this monomorphism. The adjoint mapping $J^{\prime}$ is a strong homomorphism if and only if $\left(E, \mathfrak{T}_{1}\right)$ is distinguished. In that case $J^{\prime \prime}$ is also a strong monomorphism, and $J^{\prime \prime}$ has a weakly closed image, while the equalities $J(E)^{\prime \prime}=J(E)^{\perp \perp}=\left(\operatorname{Ker} J^{\prime}\right)^{\perp}=J^{\prime \prime}\left(E^{\prime \prime}\right)$ are valid by virtue of the proposition d) of Theorem 17. By virtue of the proposition c) of Theorem 18 we have $\left(J(E)^{\prime \prime}, \beta\left(\left(F^{\prime} / \operatorname{Ker} J^{\prime}\right)^{\prime}, J(E)^{\prime}\right)=\left(J^{\prime \prime}\left(E^{\prime \prime}\right), \beta\left(F^{\prime \prime}, F^{\prime}\right) \cap J^{\prime \prime}\left(E^{\prime \prime}\right)\right)\right.$. If we consider as $\left(E, \mathfrak{T}_{1}\right)$ the distinguished Fréchet space whose bidual space is not distinguished [28], then $J^{\prime \prime \prime}$ will no longer be a strong homomorphism.

Let now $(E, \mathfrak{T})$ be the nondistinguished Fréchet space from [3]. As it is known, in that case $J^{\prime}$ is not a strong homomorphism, but $J^{\prime \prime}$ is a strong monomorphism with a strongly but not weakly closed image. Indeed, by virtue of the properties of the monomorphisms $J$ and $J^{\prime \prime}$ we have $J(E)^{\prime \prime}=\left(J(E)^{\prime}, \beta\left(J(E)^{\prime}, J(E)\right)\right)^{\prime}=$ $\left(F^{\prime} / \operatorname{Ker} J^{\prime}, \beta\left(J(E)^{\prime}, J(E)\right)\right)^{\prime} \subset\left(F^{\prime} / \operatorname{Ker} J^{\prime}, \beta\left(F^{\prime}, F\right) / \operatorname{Ker} J^{\prime}\right)^{\prime}=\left(\operatorname{Ker} J^{\prime}\right)^{\perp}=$ $\overline{J^{\prime \prime}\left(E^{\prime \prime}\right)}$. Moreover, this inclusion is proper since $E^{\prime}=F^{\prime} /$ Ker $J^{\prime}$ is bornological in the quotient topology $\beta\left(F^{\prime}, F\right) / \operatorname{Ker} J^{\prime}=\beta\left(F^{\prime}, F\right)^{\times}$, while in the topology $\beta\left(E^{\prime}, E\right)$ it is not such since by virtue of [3] there exists, on $E^{\prime}$, a linear functional which is not strongly continuous but bounded on each bounded set, i.e., continuous in the quotient topology.

Example 5. Let now $(F, \mathfrak{T})=s \cdot \lim _{\rightarrow}\left(F_{n}, \mathfrak{T}_{n}\right)$ be a strict $(L F)$-space. Then the space $(F, \mathfrak{T})$ is isomorphic to the quotient space $\bigoplus_{n \in N} F_{n} / H$ of the sum $E=$ $\bigoplus F_{n}$ with respect to the closed subspace $H$ spanned on elements of the form ${ }_{n \in N}-I_{n m} x$, where $x \in F_{n}, I_{n m}: F_{n} \rightarrow F_{m}$ is the identical mapping $(n \leq m)$. Let $k: E \rightarrow E / H=s \cdot \lim \left(F_{n}, \mathfrak{T}_{n}\right)$ be a canonical homomorphism. Then $k$ is a strong homomorphism by virtue of the Corollary of Theorem 2 , while by virtue of the Corollary of Theorem $6 k$ is a $\mathfrak{T}_{k}$-homomorphism. The adjoint mapping $k^{\prime}$ is a strong homomorphism. Indeed, each bounded set of the quotient space is contained in some $F_{n}$ and is therefore contained in the canonical image of some bounded set from $E$. This equivalent to the fulfilment of the conditions of Theorem 10. 
By an analogous reasoning one can prove that $k^{\prime}$ is a $\mathfrak{T}_{k^{-}}$and $\mathfrak{T}_{c}$-homomorphism. It is more difficult to establish whether the second adjoint mapping $k^{\prime \prime}$ is a strong homomorphism. By virtue of the proposition c) of Theorem $18 k^{\prime \prime}$ is a strong homomorphism if and only if $\left((E / \operatorname{Ker} k)^{\prime \prime}, \beta\left((E / \operatorname{Ker} k)^{\prime \prime},(E / \operatorname{Ker} k)^{\prime}\right)\right)=$ $\left(E^{\prime \prime} / \operatorname{Ker} k^{\prime \prime}, \beta\left(E^{\prime \prime}, E^{\prime}\right) / \operatorname{Ker} k^{\prime}\right)$, i.e., $\left(F^{\prime \prime}, \beta\left(F^{\prime \prime}, F^{\prime}\right)\right)=\left(E^{\prime \prime} / \operatorname{Ker} k^{\prime \prime}, \beta\left(E^{\prime \prime}, E^{\prime}\right) /\right.$ $\left.\operatorname{Ker} k^{\prime \prime}\right)$. Since $\left(E^{\prime \prime}, \beta\left(E^{\prime \prime}, E^{\prime}\right)\right)=\bigoplus_{n \in N}\left(F_{n}^{\prime \prime}, \beta\left(F_{n}^{\prime \prime}, F_{n}^{\prime}\right)\right)$, this is equivalent to that the space $\left(F^{\prime \prime} \beta\left(F^{\prime \prime}, F^{\prime}\right)\right)$ is barrelled or bornological i.e., that $\left(F^{\prime \prime}, \beta\left(F^{\prime \prime}, F^{\prime}\right)\right)=$ $s \cdot \lim _{\rightarrow}\left(F_{n}^{\prime \prime}, \beta\left(F_{n}^{\prime \prime}, F_{n}^{\prime}\right)\right)$ sence [3]. The example of a strict $(L F)$-space constructed in $[\overrightarrow{29}]$ shows that $\left(F^{\prime \prime}, \beta\left(F^{\prime \prime}, F^{\prime}\right)\right)$ is not always barrelled and bornological, i.e., $k^{\prime \prime}$ is not always a strong homomorphism.

A similar approach to the investigation of homomorphisms in the case of various topologies in locally convex spaces can also be found in [30] and [31].

\section{ACKNOWLEDGEMENT}

The author wishes to express his gratitude to the referee for useful remarks.

\section{REFERENCES}

1. J. Dieudonné, La dualité dans les espaces vectoriels topologiques. Ann. Sci. École Norm. Supér. 59(1942), 107-139.

2. J. Dieudonné and L. Schwartz, La dualité dans les espaces (F) et (LF). Ann. Inst. Fourier (Grenoble), 1949/50, 1, 61-101.

3. A. Grothendieck, Sur les espaces (F) et (LF). Summa Brasil Math. 3(1954), 57-123.

4. G. Köтнe, Die Bildräume abgeschlosener operatoren. J. Reine Angew. Math. 238(1968), 110-111.

5. G. Köthe, Homomorphismen von (F)-Räumen. Math. Z. 84(1964), 219-221.

6. F. Browder, Functional analysis and partial differential equations. I. Math. Ann. 138(1959), 55-79. II. Math. Ann. 145(1962), 81-220.

7. V. S. Retakh, On the adjoint homomorphism of a locally convex space. (Russian) Fuktsional. Anal. i Prilozhen. 3(1969), No. 4, 63-71.

8. V. P. Palamodov, Topological methods in the locally convex spaces theory. (Russian) Uspekhi Mat. Nauk 26(1971), No. 1, 1-65.

9. K. Floret, and V. B. Moscatelli, On basis in strict inductive and projective limits of locally convex spaces. Pacific J. Math. 119(1985), No. 1, 103-113.

10. G. Köтне, Topological vector spaces. II. Springer-Verlag, Berlin, Heidelberg, N. Y., 1979.

11. H. Schaefer, Topological vector spaces. Macmillan Co., New York, London, 1966; Russian transl.: Mir, Moscow, 1971.

12. S. Prössdorf, Einige Klassen singulärer Gleichungen. Akademie-Verlag, Berlin, 1974; Russian transl.: Mir, Moscow, 1979.

13. D. N. ZaRnadze, Reflexivity and best approximations in Fréchet spaces. (Russian) $I z v$. Akad. Nauk SSSR Ser. Mat. 44(1980), No. 4, 821-831; English transl.: Math. USSR Izv. 17, No. 1 (1981), 87-94. 
14. D. N. ZarnadZe, On strictly regular Fréchet spaces. (Russian) Mat. Zametki 31(1982), No. 6, 899-908; English transl.: Math. Notes 31(1982), 454-458.

15. D. A. RaYkov, Completely continuous spectra of locally convex spaces. (Russian) Trudy Moskov. Mat. Obshch. 7(1958), 413-438.

16. K. Floret, Über den Dualraum eines lokalkonvexen Unterräumes. Arch. Math. 25(1974), 646-648.

17. D. N. Zarnadze, On some topological and geometrical properties of Fréchet-Hilbert spaces. (Russian) Izv. Ross. Akad. Nauk Ser. Mat. 56(1992), No. 5, 1001-1020; English transl.: Russian Acad. Sci. Izv., Math. 41(1993), 273-288.

18. S. Dierolf and D. N. Zarnadze, A note on strictly regular Fréchet spaces. Arch. Math. 42(1984), 549-556.

19. J. Metafune and V. B. Moscatelli, Quojection and prequojections. Advances in the thoery of Fréchet spaces. Proc. NATO Adv. Res. Workshop, Istanbul/Turkey 1988, NATO ASI Ser. C287, 235-254, Kluwer Acad. Publ. Dordrech, 1989.

20. G. Vogt, On two problems of Mitiagin. Math. Nachr. 141(1989), 13-25.

21. G. Köthe, Topological vector spaces, I. Springer-Verlag, Berlin, Heidelberg, N. Y., 1983.

22. O. G. Smolyanov, On the topology of the spaces $D$ and $D^{\prime}$. (Russian) Vestnik Moskov. Univ. Ser. I Mat. Mekh. 1984, No. 1, 66-69.

23. V. S. Vladimirov, Generalized functions in mathematical physics. (Russian) Nauka, Moscow, 1979.

24. V. V. Zharinov, Compact families of locally convex spaces and FS and DFS spaces. Uspekhi Mat. Nauk 34(1979), No. 4, 97-131.

25. V. S. Petakh, On subspaces of a countable inductive limit. (Russian) Dokl. Akad. Nauk SSSR 194(1970), No. 6, 1277-1279.

26. I. Amemiya, Some examples of $(F)$ - and $(D F)$-spaces. Proc. Japan Acad. 33(1957), 169-171.

27. M. Valdivia, A class of quasi-barrelled $(D F)$-spaces which are not bornological. Math. Z. 136(1974), 249-251.

28. J. Bonet, S. Dierolf and C. Fernandez, The bidual of a distinguished Fréchet space need not be distinguished. Arch. Math. 57(1991), No. 5, 475-478.

29. J. Bonet and S. Dierolf, A note on a biduals of strict $(L F)$-spaces. Results Math. 13(1988), 23-32.

30. D. N. ZARnADZE, On homomorphisms of locally convex spaces and their adjoint mappings. (Russian) Trudy Inst. Vychisl. Mat. Akad. Nauk Gruz. SSR 26(1986), No. 1, $65-77$.

31. S. Dierolf and D. N. Zarnadze, On homomorphisms between locally convex spaces. Note Mat. 12(1992), 27-41.

(Received 5.06.2000; revised 25.03.2001)

Author's address:

N. Muskhelishvili Institute of Computational Mathematics

Georgian Academy of Sciences

8, Akuri St., Tbilisi 380093

Georgia

E-mails: zarnadze@compmath.acnet.ge,zardav44@hotmail.com 